\title{
Real-time imaging of multivesicular body-plasma membrane fusion to quantify exosome release from single cells
}

Maarten P. Bebelman ${ }^{1,2}$, Philippe Bun ${ }^{3,4}$, Stephan Huveneers ${ }^{5}$, Guillaume van Niel ${ }^{4}$, D. Michiel Pegtel ${ }^{1}$, Frederik J. Verweij ${ }^{1,4}$

${ }^{1}$ Amsterdam UMC, Vrije Universiteit Amsterdam, Department of Pathology, Cancer Center Amsterdam, de Boelelaan 1117, Amsterdam, The Netherlands.

2 Division of Medicinal Chemistry, Amsterdam Institute for Molecules Medicines and Systems, VU University Amsterdam, The Netherlands.

${ }^{3}$ Neurlmag Tech Core Facility, Institute of Psychiatry and Neurosciences of Paris, INSERM U1266, Universite Paris Descartes.

${ }^{4}$ Institute for Psychiatry and Neuroscience Paris, Hopital Saint-Anne, Université Descartes, INSERM U1266, Paris 75014, France.

${ }^{5}$ Amsterdam UMC, University of Amsterdam, location AMC, Department of Medical Biochemistry, Amsterdam Cardiovascular Sciences, Amsterdam, The Netherlands.

Correspondence to:

D. Michiel Pegtel: d.pegtel@amsterdamumc.nl, tel: +31 (0)20444 4052

Frederik J. Verweij: frederik.verweij@inserm.fr, tel: +33 140789240, fax: +33 140789204

KEYWORDS extracellular vesicle, exosome, multivesicular bodies, multivesicular body, MVB, CD63, CD81, CD9, pHluorin, pHuji, CD63-pHluorin, tetraspanin, cell biology, total internal reflection microscopy, TIRF, live cell imaging, time-lapse

EDITORIAL SUMMARY This protocol describes a collection of $\mathrm{pH}$-sensitive fluorescent reporters that can be used for real-time dual-colour imaging of exosome release from single cells. The authors provide detailed instructions for TIRF imaging and automated data analysis.

TWEET A new protocol for real-time quantitative imaging of exosome release from cells using $\mathrm{pH}$ sensitive fluorescent reporters.

COVER TEASER Real-time fluorescence imaging of exosome release

Up to four primary research articles where the protocol has been used and/or developed:

1. Verweij, F. J. et al. Quantifying exosome secretion from single cells reveals a modulatory role for GPCR signaling. J Cell Biol, doi:10.1083/jcb.201703206 (2018). 


\begin{abstract}
Exosomes are small 40-150 nm-sized extracellular vesicles implicated in cellular homeostasis and cellcell communication. Exosomes can be secreted in bulk upon cell-extrinsic and -intrinsic signals that cause multivesicular body (MVB) fusion with the plasma membrane (PM). However, research on the regulation of exosome release is hampered as current methods fail to capture the dynamics of exosome release. Here, we describe how live imaging with tetraspanin-based $\mathrm{pH}$-sensitive fluorescent reporters can quantify the MVB-PM fusion rate of single cells. Our approach enables identification of exogenous stimuli, signaling pathways, fusion complexes, and can map sub-cellular sites of fusion events. Additionally, dual-color imaging can be used to assess simultaneous cargo release by PM fusion of MVBs. This protocol describes the complete imaging experiment, consisting of transient expression of tetraspanin-reporters ( 2 days), live cell (dual-color) Total Internal Reflection Fluorescence (TIRF) microscopy (30-60 minutes per condition) and semi-automatic image analysis using a newly developed ImageJ macro ( \pm 30 minutes per condition).
\end{abstract}

\title{
Introduction
}

\section{Development of the protocol}

Late-endosomes, also referred to as multivesicular bodies (MVBs), contain small intraluminal vesicles (ILVs) enriched in subsets of lipids, proteins and nucleic acids. While the majority of MVBs are destined to fuse with lysosomes to degrade their contents ${ }^{1}$, some MVBs fuse with plasma membrane (PM) instead, releasing their ILVs into the extracellular space ${ }^{2}$. Once released, these ILVs - now called exosomes - can partake in various physiological and pathophysiological processes, including development, angiogenesis, immune regulation, trophic support, cancer progression, and development of metabolic, cardiovascular and neurodegenerative diseases ${ }^{3-7}$.

While the functions of exosomes post-secretion are intensely studied ${ }^{2,8}$, our knowledge of factors modulating the release of exosomes remains sparse, precluding more definitive tests on their physiological relevance in in vivo systems. This knowledge-gap is mainly due to the lack of methods that can monitor direct, real-time effects of potential modulators on MVB biogenesis and MVB-PM fusion. Instead, indirect, post-secretion-based types of examination are typically used, which depend on isolation by differential ultra-centrifugation and/or size-exclusion chromatography (SEC) prior to downstream analysis. A wide variety of extracellular vesicle (EV) analysis methods have been described, that are generally deployed to profile (sub-)populations of exosomes or characterize exosomal content ${ }^{9-12}$. However, these techniques are not well suited to study factors influencing the dynamics of secretion, as the extended time periods needed to acquire sufficient material allow for secondary effects of treatments and/or negative-feedback loops to occur. Furthermore, these techniques fail to distinguish MVB-derived exosomes from small PM-derived microvesicles that are indistinguishable from exosomes due to overlapping biogenesis mechanisms and biophysical properties ${ }^{13}$.

In order to visualize exosome release events, our group and Sung et $\mathrm{al}^{16}$ independently developed constructs in which the exosome-associated tetraspanin (TSPAN) CD63 was fused to a pH-sensitive green fluorescent protein pHluorin ${ }^{14,15}$. Using immuno-EM, we demonstrated that our CD63-pHluorin 
fusion-protein, like endogenous CD63, is highly enriched in ILVs. Because the pHluorin-moiety faces the MVB lumen, CD63-pHluorin fluorescence is quenched as a consequence of the low $\mathrm{pH}$ in the $M B^{17}$. Upon MVB-PM fusion, the $\mathrm{pH}$ in the MVB lumen is immediately neutralized, resulting in a sudden increase in fluorescence of CD63-pHluorin that can be detected using live cell imaging. Sung et al. used their CD63-pHluorin to visualize exosome secretion at $0.03-0.17 \mathrm{~Hz}$ in migrating cells, showing fluorescence at sites preceding adhesion formation ${ }^{16}$. We placed the pHluorin moiety in CD63's first extracellular loop, 7 amino acids upstream relative to the construct by Sung et $\mathrm{al}^{16}$. The combination of this construct with high-speed imaging ( $\geq 2 \mathrm{~Hz}$ ) enabled us to directly visualize MVBPM fusion down to the single-cell and subcellular level at high temporal resolution (Fig. 1; Supplementary Video 1$)^{17}$. Importantly, the CD63-pHluorin reporter provides the opportunity to distinguish the secretion of MVB-derived exosomes from PM-derived EV release. In our original publication ${ }^{17}$ we demonstrated the utility of high-speed CD63-pHluorin imaging in unraveling exogenous physiological stimuli, intracellular signaling pathways, and fusion machinery involved in exosome release. Meanwhile, the high-speed CD63-pHlluorin live imaging approach has been applied by Lu et al. to validate candidates from a genome-wide CRISPR/Cas9-screen for new players in MVB exocytosis ${ }^{18}$.

Here we describe a detailed protocol for the quantification of exosome release by high-speed single cell live Total Internal Reflection Fluorescence (TIRF)-microscopy using the CD63-pHluorin reporter. Although optimized for HeLa cells, we have successfully used this protocol in a variety of adherent cell lines, primary human cells and stem cells (see Materials - 'Biological Materials'). We anticipate that this approach will enable researchers to decipher a wide range of aspects of exosome secretion at high spatiotemporal resolution.

\section{Applications of the method}

CD63-pHluorin can be used to measure the frequency and sub-cellular localization of MVB-PM fusion events for a defined sub-population of (CD63-positive) MVBs. The expression and imaging of CD63pHluorin allows for a high-resolution quantitative read-out for the real-time analysis of: exogenous stimuli, intracellular signaling pathways, and fusion/SNARE complexes implicated in CD63+ exosome release, as well as their immediate post-fusion fate as described in our initial publication ${ }^{17}$. The CD63pHluorin-based live imaging approach can be combined with small molecule screening and siRNAmediated knockdown, thus enabling small targeted screens to identify modulators of MVB exocytosis. Furthermore, in our original publication we used a computer-controlled multi-valve superfusion system to assess the effect of various small molecules (including histamine) on the MVB-PM fusion frequency in HeLa cells during image acquisition ${ }^{17}$. In the absence of a superfusion system, ligands can also be added directly to the cell culture medium. However, without perfusion, this type of stimulation can only be used once per well or coverslip and care must be taken not to disturb the cell when adding the ligand to the culture medium.

Exosomes are highly heterogeneous, and not all of them carry $\mathrm{CD}^{2} 3^{9}$. To address this heterogeneity, we developed $\mathrm{pH}$-sensitive fluorescent reporters based on additional EV-associated TSPANs CD81 and $\mathrm{CD} 9$ that were tagged with a green (pHluorin) or red ( $\mathrm{pHuji}$ ) $\mathrm{pH}$-sensitive fluorescent protein. These reporters can be used individually, or in combination using dual-color imaging (Fig. 2a,b; Supplementary Video 2). In combination with the $\mathrm{pH}$-sensitive TSPAN-reporters, $\mathrm{pH}$-insensitive fluorescent proteins can be used to tag TSPANs or other exosomal cargoes of interest (Fig. 2a,b; 
Supplementary Video 3). Of note, positioning of the pHluorin tag at the cytoplasmic side of proteins is also possible, as we previously showed for a C-terminally tagged version of CD63-pHluorin (Fig. 2a,b; Supplementary Video 4; Ref ${ }^{17}$ ). Since the exosomal membrane is permeable for protons ${ }^{19-21}$, pHluorin molecules facing the exosome/ILV lumen will be quenched in the MVB, but become fluorescent upon MVB-PM fusion. The possibility to place the pHluorin at the cytoplasmic side provides flexibility in the design of the tagged protein of interest with respect to domains that regulate the intracellular trafficking or its interaction with crucial partners. Care should be taken, however, as the pHluorin molecules that are located at the cytoplasmic side of the limiting membrane (LM) will be fluorescent even before MVB-PM fusion. This may increase background, especially for proteins that have a (relatively) high LM-to-ILV localization ratio, such as LAMP-1. The LM-to-ILV ratio of a protein can be determined by immuno-electron microscopy ${ }^{22}$.

The CD63-pHluorin method is compatible with stable (lentiviral-transduced) cell lines ${ }^{18}$. This provides the user with stable expression levels and will improve reproducibility, which is beneficial in targeted screens. In our hands, MVB-PM fusion events could be detected 6 weeks after the generation of a stable CD63-pHluorin expressing HeLa cell line (Supplementary Video 5). However, some applications, such as visualization of exosome deposition by migrating cells, might require higher CD63-pHluorin expression levels. To this end, Sung et al. recently developed a CD63-pHluorinM153R mutant that offers increased stability and higher expression levels of the fusion protein, which could be used when the brightness of regular CD63-pHluorin is not sufficient ${ }^{23}$.

One facet we did not explore in full detail and that may provide additional biological insight is the mapping of the sub-cellular localization of MVB-PM fusion events. Indeed, exosomes can be released in a polarized fashion, for instance in the context of an (antigen-induced) immunological synapse between $\mathrm{T}$ - and $\mathrm{B}$-cells $\mathrm{s}^{24}$, on the front end of migrating cancer cells $\mathrm{s}^{23}$, or on the apical and basolateral side of cells, as shown for intestinal epithelial cells ${ }^{25}$. These exosomes might also very well differ in their respective composition ${ }^{25}$. At the basolateral side of cells, exosome secretion can be enhanced at invadopodia ${ }^{26}$. Interestingly, whereas initial flat lamellipodia of HT1080 cells are not enriched in CD63pHluorin fluorescence, they do show local concentrations of fluorescence in the minutes preceding adhesion assembly, suggestion local exosome release ${ }^{16}$. It could thus be of interest to use CD63pHluorin (or other cargoes) to image MVB-PM fusion events at high speed for detailed analysis of MVB exocytosis at these dynamic membrane structures.

Another exciting application of the reporters is the in vivo analysis of exosome release, which will allow the study of exosome release kinetics in a full physiological context. Indeed, we recently used CD63-pHluorin expressed in zebrafish to detect in vivo MVB-PM fusion events with similar intensity and duration as described in vitro ${ }^{27}$. Currently, this type of analysis is limited to relatively flat cells that are ideally oriented parallel with the imaging plane. The full potential of this application therefore will only be realized when using imaging techniques that combine a fast acquisition speed with a high $(x, y, z)$ resolution and depth while maintaining low phototoxicity. The use of CD63-pHluorin also enables the tracking of single EVs after their release in biological fluids ${ }^{27}$. Compared to CD63-GFP, the use of the $\mathrm{pH}$-sensitive CD63-pHluorin is highly advantageous as most of the intracellular CD63pHluorin in producing cells, or in the endocytosed EVs in the late endosomal compartments of recipient cells, is quenched, thus increasing the sensitivity for extracellular (exosomal) CD63-pHluorin. Importantly, CD63-pHluorin on the cell surface or in non-acidic compartments might still cause high 
background in tissues overexpressing CD63-pHluorin. Similarly, endocytosed EVs that reside in early endocytic compartments of recipient cells will initially be fluorescent, until the endosomes mature and their $\mathrm{pH}$ decreases. To visualize EV endocytosis, CD63-pHluorin can be combined with a $\mathrm{pH}$ insensitive red fluorescent protein ${ }^{23}$ or the V-ATPase inhibitor bafilomycin A1, which neutralizes the $\mathrm{pH}$ of the late endosomal compartments in recipient cells ${ }^{27}$. Finally, the use of a pHluorin tag placed in the extracellular loop of the TSPANs also offers the advantage to combine live cell imaging with immunoelectron microscopy or anti-GFP immunoprecipitation, followed by RNA sequencing, proteomics or western blot, notably in model organisms where antibodies for exosomes-markers are lacking ${ }^{27}$.

\section{Comparison with other methods}

Over the years, a wide variety of EV quantification methods have been developed that have been instrumental in the study of exosome biogenesis, but also in characterizing subgroups of EVs and their respective cargoes ${ }^{12,28}$. The most direct way to quantify exosome release is by isolating the exosomes from cell culture supernatant, followed by electron microscopy. This technique allows accurate quantification and sizing of EVs but has the disadvantage of being labor-intensive and low-throughput. Therefore, other direct particle quantification methods have been developed, including nanoparticle tracking analysis (NTA) and high-resolution flow cytometry. Currently, NTA is one of the most widely used techniques for EV quantification ${ }^{12}$. This microscopy-based technique detects EVs by their light scatter during laser-illumination and determines vesicle size based on their Brownian motion in suspension. Unfortunately, there are a number of factors that limit the use of NTA for studying exosome release. First of all, NTA has a lower detection limit of \pm 70 nanometer, causing smaller exosomes to be underrepresented or absent from analysis ${ }^{29}$. Furthermore, accurate EV quantification by NTA requires EV concentrations that are often not reached without pre-isolation of the vesicles from the culture supernatant. However, EV isolation methods, such as differential ultracentrifugation, can cause EV aggregation and co-isolate lipoparticles and/or nucleic acid-protein aggregates' that contribute to NTA concentration measurements ${ }^{30-32}$. Advanced flow cytometry is another promising technique that has the potential to count, size and analyze the surface protein content of single EVs ${ }^{10}$. Currently, this technique seems most suitable for analysis of larger EV subsets, since the lower end of the exosome size range $(40-100 \mathrm{~nm})$ still falls below the detection limit of most flow cytometers ${ }^{29}$.

MVB-derived exosomes and small PM-derived vesicles, generally called microvesicles, actually have overlapping size ranges and similar biophysical properties. This makes it impossible to selectively quantify exosome secretion using single particle tracking techniques. In fact, differentiating between these two subtypes by size and/or density is impossible. To overcome this, western blot for EVassociated endosomal markers (e.g. CD63, CD81, ALIX) is frequently used to determine relative changes in exosome release. However, several studies performing extensive biochemical separation of the EV fraction have shown a significant overlap in protein content between EV subtypes, suggesting that these markers are not exclusive to MVB-derived exosomes ${ }^{9,33,34}$.

The main disadvantage of using the currently available exosome quantification methods, however, is that they require concentrated exosome preparations for accurate analysis. Consequently, EVs are generally collected from the culture medium of millions of cells over a time-course of 24-72 hours. By contrast, the CD63-pHluorin approach allows the study of exosome release in real-time from a single 
cell. This enables the detection of subtle and temporary changes in exosome release that might not be picked up in long-term culture due to receptor downregulation or other negative feedback on the signaling pathway. In addition, it permits the distinction of direct from secondary effects when using drugs/compounds that, on the long run, affect cellular health in general or could otherwise introduce secondary effects on exosome release.

In conclusion, the majority of the aforementioned alternative techniques facilitate a more precise characterization of EV content and allow quantification of population-based (changes in) EV release over extended time-periods. On the other hand, the high-speed CD63-pHluorin imaging approach explained here provides direct, real-time insight in exosome release at the single cell level. This complements the traditional, more indirect biochemical approaches to study the molecular mechanisms of exosome release.

\section{Limitations of the protocol}

The application of CD63-pHluorin as tool to study exosome release and deposition from single cells have been reported in multiple independent publications ${ }^{16,18,23,35}$, yet TSPAN-reporters have several limitations that are inherent to the technique. First, the high-speed CD63-pHluorin live imaging approach we describe here reports MVB-PM fusions rather than actual exosome exocytosis. In our original publication we showed that CD63-pHluorin fusion activity correlates with the amount of secreted CD63 in the EV fraction as assessed by western blot ${ }^{14}$. Yet, under certain conditions exosome release might not be directly reflected by MVB-fusion rate for instance when cell manipulations directly affect CD63 sorting from the LM into ILVs. Of note, the CD63-C-term-pHluorin construct can be used to visualize a defect in ILV sorting of CD63. As described in the 'Applications of the method', CD63-C-term-pHluorin-containing MVBs are already visible before exocytosis, but show a burst of additional fluorescence when the MVB fuses with the PM and the inside of the ILVs becomes neutral ${ }^{17}$. In case of defective sorting of CD63-C-term-pHluorin, these additional bursts of fluorescence will not be observed. Second, CD63-pHluorin only reports the release of CD63-positive exosomes, meaning that EV subpopulations devoid of the specific reporter will inevitably not be visualized ${ }^{9}$, although this can also be seen as a strength for specificity of the approach. Choosing a different TSPAN- (or other exosome specific cargo) pHluorin-reporter, as detailed in the experimental design section, may expand the applicability of the approach. Importantly, imaging of CD63-pHluorin ignores generation of MVs or exosome-like vesicles (e.g. ARMMs) budding from the PM, or release of small nonmembranous particles such as exomeres ${ }^{34,36}$, which again, depending on the scientific question, could be viewed as both a limitation or strength ${ }^{37}$.

A limitation of TIRF-based imaging is the requirement of a relatively flat plasma membrane in close proximity to the coverslip, effectively restricting the approach to adherent cells. Other imaging techniques, like spinning-disk microscopy, could in theory be used to visualize fusion events at higher focal-planes. However, due to a lower signal-to-noise ratio compared to TIRF microscopy, combined with the lower total membrane surface area at higher focal planes, fusion spots will be less easily detected. In addition, we found that there is some intercellular variation in MVB-PM fusion activity. Therefore, measuring significant changes in MVB-PM fusion activity between conditions requires the imaging of multiple cells (we advise at least 10 cells per condition). As both the imaging and the analysis of fusion activity require 30-60 minutes per condition, this approach in its current format will be mostly apt for small targeted screens. 


\section{Experimental design}

The procedure described here consists of three stages: preparation of the cells (Steps 1-9), live cell imaging (Steps 10-28) and the image analysis (Steps 29-37). To detect MVB-PM fusion, the cells are transiently transfected using $\mathrm{pH}$-sensitive fluorescent reporters. These cells are then subsequently imaged using live cell TIRF microscopy, and MVB-PM fusion events can be quantified using the Analyzer of MVB Exocytosis (AMvBE) macro (Supplementary Software 1).

Choice of $\mathrm{pH}$-sensitive fluorescent reporters. This protocol for the imaging of MVB-PM fusion in living cells is based on the expression of EV-associated tetraspanins (TSPANs) fused to $\mathrm{pH}$-sensitive fluorescent proteins. In our original publication we focused on CD63 fused to Superecliptic pHluorin ${ }^{14}$. This green fluorescent protein has excellent $\mathrm{pH}$-sensitivity and is almost completely quenched at the acidic $\mathrm{pH}$ of multivesicular bodies ${ }^{14,15,17}$. We have fused the pHluorin moiety in the first extracellular loop of CD63 between Gln36 and Leu37. Furthermore, the construct developed by Sung et al., in which the pHluorin moiety is placed 7 amino acids away in the first extracellular loop, can also be used to visualize exosome secretion as we have no reason to assume that it behaves dissimilar under highspeed imaging conditions ${ }^{16}$. In addition to CD63-pHluorin, we developed reporters based on CD81 and CD9, two other TSPANs that are frequently used as EV marker.

To complement the green pHluorin, a number of red $\mathrm{pH}$-sensitive fluorescent proteins have been developed, such as pHoran4, pHTomato and $\mathrm{pHuji}^{38}$. Here we chose to use pHuji, based on its pKa of 7.7, its $\mathrm{pH}$-sensitivity and its emission wavelength of $598 \mathrm{~nm}$, which makes it possible to combine it with pHluorin for multi-channel imaging without fluorescence bleed-through. Although a TSPAN-pHuji can be used to detect MVB-PM fusion, the fold increase in fluorescence intensity upon fusion is higher for CD63-pHluorin compared to CD63-pHuji (Fig 2c), which is in line with the difference in $\mathrm{pH}$ sensitivity between pHluorin and $\mathrm{pHuji}^{38}$. This results in a slightly higher sensitivity for fusion events with pHluorin as compared to pHuji. Therefore, we recommend that detection of MVB-PM fusion in single-color is performed using the TSPAN-pHluorin reporters.

Simultaneous dual-color imaging of cells co-expressing TSPAN-pHluorin and other cargo tagged with red fluorescent proteins, such as the TSPAN-pHuji reporters, can be a powerful tool to assess joint cargo loading and release by PM-fusing MVBs (Fig 2a,b; Supplementary Video 2). Furthermore, coexpression of a TSPAN tagged with a $\mathrm{pH}$-independent red fluorescent proteins can be used to track MVBs before fusion (Fig 2a,b; Supplementary Video 3). Similarly, the use of a CD63 construct dualtagged with pHluorin and $\mathrm{mScarlet}$ has recently been employed to visualize MVBs before fusion and to study exosome endocytosis ${ }^{23}$. MVB cargo molecules might be tagged at different locations regarding to their membrane orientation. Dependent on the type and the location of the fluorescent label, the theoretical fusion profile will differ (Fig. 2a,b).

Preparation of the cells. TIRF microscopy requires that the cells are plated on coverglass with a thickness matching the objective that is used. We routinely use Nunc Lab-Tek chambered coverglass (borosilicate glass, thickness \#1), but we have previously used $35 \mathrm{~mm} \# 1.5$ glass FluoroDishes or 18 $\mathrm{mm}$ round \#1 glass coverslips that were mounted in an imaging chamber. HeLa cells will adhere to the glass without the need for any additional coating. However, we noticed that coating the coverglass 
with poly-L-lysine increases the contact surface of the cell with the coverslip, resulting in a more even illumination of the plasma membrane.

The current protocol relies on transient transfection of the cells with CD63-pHluorin or one of the other reporters. For the transfection of HeLa cells, we routinely use lipofectamine2000 (ThermoFisher Scientific), but other transfection reagents such as JetPrime (PolyPlus transfection) might be used as well. Instead of transfection, a lentiviral transduction protocol can be used to express the reporters in cell lines or primary cells where transfection is inefficient. Lentiviral transduction also allows for the generation of stable TSPAN-reporter cell lines through integration of the reporters in the genomic DNA. We have cloned all TSPAN-reporters presented here also in the pLenti6.3/TO/V5-DEST vector (available upon request) that can be used with the ViraPower ${ }^{\mathrm{TM}}$ HiPerform $^{\mathrm{TM}}$ T-Rex $^{\mathrm{TM}}$ Gateway $^{\circledR}$ Expression System (ThermoFisher Scientific). Of note, CMV-promoter driven overexpression of proteins can lead to a growth disadvantage over time. This will result in selection of cells that express the TSPAN-reporters at (undetectable) low levels from the original polyclonal lentivirus transduced population. To avoid this, cells can be co-transduced with the pLenti3.3/TR repressor (Invitrogen) to allow tetracyclin-regulated expression of the TSPAN-reporters.

Imaging set-up. To image the MVB-PM fusion events one can use any fast, high-resolution objectivebased TIRF microscopy set-up. TIRF microscopy allows for selective illumination of the basal plasma membrane and thus provides optimal signal-to-noise ratio as well as low phototoxicity. In addition to TIRF microscopy, MVB-PM fusion events can be detected using spinning-disc confocal microscopy. However, the number of detected fusion events by spinning-disc confocal is lower than with TIRF due to reduced signal-to-noise ratio compared to TIRF. This protocol describes the use of a Nikon Eclipse TI-E, equipped with an APO TIRF 60x/1.49 Oil objective (Nikon) and a sCMOS camera (Zyla; Andor). The protocol has also successfully been performed using a Zeiss Axiovert 200M microscope equipped with an Alpha Plan-Fluar 100x/1.45 Oil TIRF objective (Zeiss) and an EMCCD camera (CASCADE; Roper Scientific) and with a Nikon Eclipse TI-E equipped with a CFI Apochromat 100x/1.49 Oil TIRF objective (Nikon) and an EMCCD camera (Evolve 512; Photometrix). For optimal quality of the imaging data, the coverslip thickness should be matched to the objective that is used.

For dual-color imaging of MVB-PM fusion events we use a dual band filter cube combined with a dichroic beamsplitter and a dual-camera system. This set-up allows for simultaneous imaging of both a TSPAN-pHluorin and a TSPAN-pHuji reporter at high speed. Optionally, an optical image-splitter can be used to simultaneously image two colors on a single camera, although this will result in a reduced field of view. In theory, a single camera system combined with distinct green and red filter cubes can also be used. However, for this the system needs to continuously switch between filter cubes, which drastically reduces the imaging speed and prevents imaging at the minimal frequency of $2 \mathrm{~Hz}$ that is needed to capture the fast fusion events.

Imaging MVB-PM fusion events. With high-speed imaging, fusion of a CD63-pHluorin-containing MVB with the plasma membrane can be observed as a rapid localized increase in fluorescent intensity, followed by a slow decrease of the signal upon dissipation of the secreted exosomes. Interestingly, the signal duration for CD63-pHluorin MVB-PM fusion events is significantly longer than that observed for PM-deposition of VAMP2-pHluorin from the limiting membrane of secretory organelles or soluble release of NPY-pHluorin. We hypothesize that entrapment of TSPAN-pHluorin exosomes between cells 
and the coverslip, sticking of the exosomes to the PM, or exosome-extracellular matrix interactions ${ }^{16}$ after MVB-PM fusion lies on the basis of this difference in signal duration. Whereas CD81-pHluorin shows extended signal duration similar to CD63-pHluorin, the post-fusion dynamics of most CD9pHluorin fusion events are more similar to those of VAMP2-pHluorin, suggesting that only a fraction of CD9-pHluorin fusion events report MVB exocytosis. We therefore recommend the use of CD63pHluorin or CD81-pHluorin to quantify MVB-PM fusion rate.

One of the characteristics of TIRF imaging is that the brightness of fluorescent structures increases when they are located closer to the coverslip, where the evanescent wave is generated. As a result, focal adhesions, retraction fibers and even (clusters of) exocytosed exosomes that are adherent to the coverslip ${ }^{16}$ appear as bright structures in the field of view. To distinguish these structures from intracellular non-acidic CD63-pHluorin compartments it is possible to do an acid wash using Tyrode's medium with $\mathrm{pH} 5.0^{39}$. Similarly, one can quantify the amount of acidic TSPAN-pHluorin-containing endosomes in the cell using Tyrode's solution with $\mathrm{NH}_{4} \mathrm{Cl}$, which neutralizes all acidic cellular compartments. In combination with a TIRF set-up, this will highlight the endosomes that are close to the plasma membrane.

When designing a TSPAN-pHluorin imaging experiment, it is important to realize that basal MVB-PM fusion activity (the number of fusion events per minute) is highly variable between cell lines. Therefore, the acquisition duration as well as the number of imaged cells has to be optimized for each cell line. For HeLa cells we usually require $>5$ acquisitions of separate imaging fields containing \pm 2 cells for 3 minutes per acquisition. The number of acquisitions should be increased in case the field of view is too small to capture multiple cells simultaneously. Furthermore, basal fusion activity, even in the same cell-type, is generally not the same in independent experiments. Thus, it is necessary to take along a non-treated control in each experiment when studying the effect of a certain treatment on MVB-PM fusion activity.

Transient transfection of the HeLa cells results in variable TSPAN-pHluorin expression levels. As overexpression of the TSPAN-pHluorin might result in impaired trafficking, we only select cells with moderate expression levels for imaging. The acquisition time and laser power should be set to achieve high signal-to-noise ratio, while avoiding photobleaching and phototoxicity. Furthermore, avoid saturation of the fluorescent signal in the fusion events, as this can interfere with subsequent image analysis by the Analyzer of MVB Exocytosis macro.

When considering acquisition settings, ensure that the imaging speed is fast enough $(\geq 2 \mathrm{~Hz})$ to distinguish MVB-PM fusion from vesicles with neutral $\mathrm{pH}$ approaching the plasma membrane. Furthermore, a high spatial resolution is required to allow a reliable size estimation of the fusion event and to detect potential movement of the vesicle after fusion. We routinely use a pixel sizes of $160 \mathrm{~nm}$ and $107 \mathrm{~nm}$.

Image analysis. Following image acquisition, the MVB-PM fusion activity of individual cells can be determined by counting the number of full MVB-PM fusion events that can be observed per minute. A full MVB-PM fusion event can be recognized by a sudden increase in fluorescence intensity, resulting from the instant neutralization of the $\mathrm{pH}$ of the MVB upon exocytosis and rapid movement of the secreted exosomes towards the coverslip into the TIRF field. This sudden increase is followed by an 
exponential decrease with a minimal duration of two seconds, during which the event remains in the same location (Fig 3a,b). Importantly, increases in fluorescent intensity can be detected that are not a consequence of full MVB-PM fusion, and thus do not report exosome release. Crucially, these nonproductive events can be discriminated from full MVB-PM fusion in live imaging by analysis of the fluorescent profile right before and following initial fusion (Fig. 3a,b). For example, in some cases a sudden increase in fluorescent intensity is followed by no decrease or an irregular decrease of the signal and/or lateral movement of the fluorescent signal after fusion, suggesting that the MVB is moving in the cell after neutralization. This type of events is reminiscent of so-called synaptic vesicle kiss-and-run events where the vesicle pore transiently opens and closes without fully collapsing into the plasma membrane. In the case of TSPAN-pHluorin, kiss-and-run-like fusion events might result from MVB-PM fusion with incomplete exosome secretion, or by full fusion and rapid reuptake of exocytosed exosomes. In both cases, there is no or incomplete exosome secretion and thus we exclude these kiss-and-run-like events from our analysis. Another cause for an increase in fluorescence that occurs more frequently is when a TSPAN-pHluorin-containing (transport) vesicle with neutral pH approaches the plasma membrane and moves into the TIRF field without fusing with the plasma membrane. These neutral vesicles can be distinguished from MVB-PM fusion events based on the duration of the fluorescence increase and their lateral movement in the TIRF field (Fig 3a,b). In Box 1 we provide a set of guidelines for the analysis of TSPAN-pHluorin live imaging data.

The TSPAN-pHluorin imaging procedure is based on the localization of the TSPAN-pHluorin in acidic MVBs and subsequent neutralization of these compartments by their fusion with the PM. However, a fraction of the fusion events that can be observed with the TSPAN-pHuorins, can result from PM fusion of other acidic endosomal TSPAN-positive vesicles. For example, fusion events might arise from plasma membrane deposition of CD63-pHluorin from transport vesicles or lysosomes. In our original publication we show that in HeLa cells, more than 95\% of CD63-pHluorin fusion events report exosome secretion ${ }^{17}$. As mentioned earlier, the signal duration after exocytosis of CD63-pHluorin and CD81pHluorin MVBs is significantly longer as compared to that observed for PM deposition of limiting membrane-associated VAMP2-pHluorin. This difference in signal duration allows us to discriminate between MVB-PM fusion events and the exocytosis of endosomes or lysosomes that only have TSPANpHluorin in the limiting membrane. Under the experimental conditions described in this protocol, the fusion duration of VAMP2-pHluorin fusion events is shorter than 2 seconds. Hence, we have set the minimal signal duration time for MVB-PM fusion at 2 seconds (Box 1). However, absolute fusion duration times might depend various factors, such as cell type, temperature, viscosity of the medium and coverslip coating. Therefore, it is recommended to determine the relative fusion duration of PMdeposition of VAMP2-pHluorin as compared to CD63-pHluorin in your system. Increasing the imaging frequency to $5 \mathrm{~Hz}$ might allow for a better analysis of the fast VAMP2-pHluorin fusion events.

Manual counting of the MVB-PM fusion activity based on the guidelines described in Box 1 is possible, but requires careful examination of each individual fusion event and is therefore a time-consuming process. To shorten the image analysis time, we developed a FIJI/ImageJ macro named the "Analyzer of Multivesicular Body Exocytosis (AMvBE)" (available as Supplementary Software 1). Upon opening of a TSPAN-pHluorin TIRF microscopy time-lapse with the AMvBE macro, the user can pinpoint all sudden increases in fluorescent intensity that resemble fusion events. The AMvBE macro will then track and analyze these events and test whether they comply to a number of parameters that are set by the user before analysis. Although these parameters can be changed by the user, we recommend 
the settings in Box 2 for the detection of MVB-PM fusion, which are based on the criteria as described in Box 1. After analysis, the macro provides the user with a list of "true" MVB-PM fusion events that contains data on their intensity, duration and position.

There are a number of important variables to consider when analyzing the fusion activity with AMvBE. First of all, AMvBE is developed for detecting MVB-PM fusion with CD63-pHluorin and CD81-pHluorin, and cannot be used to quantify fusion events resulting from soluble release or PM deposition. For this, other macros have previously been developed ${ }^{40,41}$. Second, the macro corrects small inaccuracies in pinpointing the events by automatic detection of the peak intensity in both space and time around the selected ROI (Fig 3c). However, this event correction in time prevents the analysis of fusion events that occur in the first or last 3 seconds of the acquisition. This should be considered when choosing the image acquisition time. Finally, drift or significant photobleaching during imaging can hamper the analysis. To avoid this, we included the option to correct for cell movement and photobleaching. However, when experiencing photobleaching we recommend lowering acquisition time and laser power to avoid both bleaching and phototoxicity, as this might affect fusion activity.

\section{Materials}

\section{Biological Materials}

- Cell lines: In the example data shown in this protocol we use HeLa cells (Sigma-Aldrich, ECACC, cat. no. 93021013, RRID:CVCL_0030). We have also successfully tested our protocol using HUVEC, HEK293T, Caski, SiHa, HCT116, Caco-2, MSC and MNT-1 cells and we anticipate it should work with most adherent cell lines and primary cells.

CRITICAL The cell lines used in your research should be regularly checked to ensure they are authentic and not infected with mycoplasma.

\section{Reagents}

Plasmids

CRITICAL: The following TSPAN-reporters are available from Addgene.

- CD63-pHluorin in pCMV-SPORT6 (Addgene ID 130901)

- CD81-pHluorin in pCMV-SPORT6 (Addgene ID 130903)

- CD9-pHluorin in pCMV-SPORT6 (Addgene ID 130905)

- CD63-pHuji in pCMV-SPORT6 (Addgene ID 130902)

- CD81-pHuji in pCMV-SPORT6 (Addgene ID 130904)

- CD9-pHuji in pCMV-SPORT6 (Addgene ID 130906)

CRITICAL: TSPAN-reporters in the pLenti6.3/TO/V5-DEST vector (for lentiviral transfection) are available upon request.

\section{Cell culture and transfection reagents}

- DMEM + GutaMAX-I (Gibco, cat. no. 61965-026)

- DMEM without phenol red (Gibco, cat. no. 31053-028)

- $10 \%$ (vol/vol) Fetal Bovine Serum (GE Healthcare Hyclone, cat. no. SV30160.03)

- Penicillin-Streptomycin-Glutamine (Gibco, cat. no. 10378-016)

- Trypsin-EDTA (Gibco, cat. no. 15400-054) 
- Opti-MEM (Gibco, cat. no. 31985-070)

- Lipofectamine 2000 (Thermo Fisher, cat. no. 11668027)

- Poly-L-lysine $0.1 \%$ (wt/vol) in $\mathrm{H}_{2} \mathrm{O}$ (Sigma-Aldrich, cat. no. P8920)

Chemicals

- $\mathrm{CaCl}_{2}$ (Sigma-Aldrich, cat. no. C7902)

CAUTION $\mathrm{CaCl}_{2}$ causes serious eye irritation. Work in a fumehood and use personal protective equipment when handling $\mathrm{CaCl}_{2}$.

- $\quad \mathrm{KCl}$ (Serva, cat. no. 26868.02)

- $\mathrm{NaCl}$ (Sigma-Aldrich, cat. no. 31434)

- $\mathrm{MgCl}_{2}$ (Sigma-Aldrich, cat. no. M9272)

- Glucose (Merck cat. no. 1.04074)

- HEPES (Sigma-Aldrich, cat. no. H4034)

- $\quad \mathrm{NH}_{4} \mathrm{Cl}$ (Riedel-de Haën, cat. no. 31107)

CAUTION NH${ }_{4} \mathrm{Cl}$ causes serious eye irritation and is harmful if swallowed. Work in a fumehood and use personal protective equipment when handling $\mathrm{NH}_{4} \mathrm{Cl}$.

- MES (2-[N-morpholino] ethane sulfonic acid) (Sigma-Aldrich, cat. no. M8250)

\section{Equipment}

- Humidified cell culture incubator, $5 \% \mathrm{CO}_{2}$

- Level 1 biosafety cabinet

- Nunc Lab-Tek chambered coverglass 8 well, borosilicate glass, thickness \#1 (ThermoFisher, cat. no. 155411)

\section{TIRF microscope}

- Nikon Eclipse Ti microscope with motorized TIRF illuminator unit (Nikon)

- Stage top incubator and cage incubator with $\mathrm{CO}_{2}$ control and humidity module (Okolab)

- Argon laser $488 \mathrm{~nm}$ (Melles Griot, model no. IMA101040ALS)

CAUTION Laser light can severely harm the eye. Ensure that laser light is always shielded from the user, and wear protective glasses while working with lasers.

- DPSS laser 594 nm (Spectra-Physics, model no. Excelsior-594-30)

- Dual-band filter cube 488/594 nm (Chroma, model no. TRF59905)

- Two-camera imaging adapter (Andor, TuCam)

- Dichroic beamsplitter (dichroic mirror: $552 \mathrm{~nm}$, emission mCherry 641/75 nm, emission GFP $520 / 35 \mathrm{~nm}$ )

- $\quad$ sCMOS camera (Andor, Zyla)

- TIRF objective, CFI Apochromat TIRF 60x/1.49 Oil (Nikon)

- Perfect Focus System (Nikon)

\section{Analysis software}

- NIS-Elements image acquisition software (Nikon)

- The most up-to-date version of the FIJI distribution of Image ${ }^{42}$ (https://imagej.net/Fiji/Downloads) supplemented with the MultiStackReg 
(http://bradbusse.net/downloads.html)

(http://bigwww.epfl.ch/thevenaz/turboreg/) plug-ins.

- The AMvBE macro (Supplementary Software 1)

\section{Reagent set-up}

\section{Full cell culture medium}

DMEM supplemented with $10 \%$ (vol/vol) FBS, $1 \mathrm{U} / \mathrm{ml}$ Penicillin G,1 mg/ml streptomycin sulfate and 2 $\mathrm{mM}$ glutamine. Store at $4{ }^{\circ} \mathrm{C}$ for up to 4 weeks.

\section{Tyrode's solution $\mathrm{pH}$ 7.4:}

$2 \mathrm{mM} \mathrm{CaCl}_{2}, 2.5 \mathrm{mM} \mathrm{KCl}, 119 \mathrm{mM} \mathrm{NaCl}, 2 \mathrm{mM} \mathrm{MgCl}_{2}, 30 \mathrm{mM}$ glucose, and $25 \mathrm{mM} \mathrm{HEPES}, \mathrm{pH}$ 7.4. Sterilize the solution using a 0.2 micron filter and store at $4{ }^{\circ} \mathrm{C}$ for up to 1 year.

\section{Tyrode's solution $\mathrm{pH}$ 5.5:}

$2 \mathrm{mM} \mathrm{CaCl}, 2.5 \mathrm{mM} \mathrm{KCl}, 119 \mathrm{mM} \mathrm{NaCl}, 2 \mathrm{mM} \mathrm{MgCl}$, $30 \mathrm{mM}$ glucose, and $25 \mathrm{mM}$ MES (2-[Nmorpholino] ethane sulfonic acid), $\mathrm{pH}$ 5.5. Sterilize the solution using a 0.2 micron filter and store at $4{ }^{\circ} \mathrm{C}$ for up to 1 year.

\section{Tyrode's solution $50 \mathrm{mM} \mathrm{NH}_{4} \mathrm{Cl}$ :}

$2 \mathrm{mM} \mathrm{CaCl}_{2}, 2.5 \mathrm{mM} \mathrm{KCl}, 119 \mathrm{mM} \mathrm{NaCl}, 2 \mathrm{mM} \mathrm{MgCl}_{2}, 50 \mathrm{mM} \mathrm{NH}{ }_{4} \mathrm{Cl}, 30 \mathrm{mM}$ glucose, and $25 \mathrm{mM}$ HEPES. Sterilize the solution using a 0.2 micron filter and store at $4{ }^{\circ} \mathrm{C}$ for up to 1 year.

\section{Poly-L-Lysine coated Lab-Tek glass chamber slides:}

Dilute the poly-L-lysine solution $10 x$ in sterile double-distilled $\mathrm{H}_{2} \mathrm{O}$ (final concentration: $0.01 \%$ (wt/vol)) and add 400 ul to each well of a Lab-Tek 8 well glass chamber slide. Incubate for 5 minutes at room temperature and remove the poly-L-lysine solution. Wash twice with double-distilled $\mathrm{H}_{2} \mathrm{O}$ and let the Lab-Tek slide dry at room temperature in the biosafety cabinet for at least 1 hour without lid. Poly-Llysine-coated Lab-Tek slides can be prepared in advance and stored under sterile conditions at room temperature $\left(21-24^{\circ} \mathrm{C}\right)$ for up to 1 year.

CRITICAL this should be performed under sterile conditions

CRITICAL scratches in the glass slide can hamper the TIRF imaging. To avoid scratching the glass bottom of the Lab-Tek slide, we recommend keeping the slide inside a $10 \mathrm{~cm}$ culture dish.

\section{Procedure}

Plating and transfection of the cells Timing 2 days, 1 hour hands-on time

CRITICAL this protocol describes the live imaging of MVB-PM fusion using the CD63-pHluorin reporter in HeLa cells. The protocol can easily be used with other TSPAN-reporters and/or applied to other adherent cells, although transient transfection of other cell types might require a different transfection protocol. Step 3-9 can be skipped when using lentiviral-transduced cells stably expressing TSPAN-reporters. 
1. HeLa cells are maintained in a T75 flask in full cell culture medium in a humidified incubator at $37{ }^{\circ} \mathrm{C}$ and $5 \% \mathrm{CO}_{2}$. To passage, remove the culture medium, rinse the T75 flask with $2 \mathrm{ml}$ PBS, add $2 \mathrm{ml}$ trypsin and incubate the cells at $37^{\circ} \mathrm{C}$ until the cells have detached (typically 3 5 minutes). After the cells have detached, add $8 \mathrm{ml}$ full medium and resuspend the cells thoroughly to obtain a single cell suspension. Split the HeLa cells two times a week at a 1:20 ratio.

2. Seed the cells at a density of $25-30 \%$ in poly-L-lysine-coated a Lab-Tek 8 well chambered coverglass and let them attach for 24 hours in a humidified incubator at $37{ }^{\circ} \mathrm{C}$ and $5 \% \mathrm{CO}_{2}$.

3. Cell transfection (Steps 3-9): Transfect the HeLa cells at 50-60\% confluency with CD63pHluorin plasmid DNA using lipofectamine 2000. In order to do so, first dilute 500 ng CD63pHluorin plasmid DNA in Opti-MEM to a total volume of $100 \mu$.

CRITICAL STEP For dual-color imaging with the TSPAN-reporters we recommend using $250 \mathrm{ng}$ of the TSPAN-pHluorin and 250 ng of the TSPAN-pHuji.

CRITICAL STEP It is critical to achieve a high transfection efficiency without inducing cell toxicity from too high overexpression of the TSPAN-reporters or the lipofectamine 2000. Increase or decrease the amount of DNA and lipofectamine 2000 when necessary. In addition to lipofectamine, other transfection reagents such as JetPrime might also be used according to manufacturer's protocol.

CRITICAL STEP we recommend doing the whole transfection procedure in a flow cabinet and incubator close to the imaging set-up. However, when cells need to be moved to an incubator close to the imaging set-up (e.g. cells are out of the incubator for more than 15 minutes) on the day of imaging, we recommend that the cells are kept in this incubator to acclimate for at least in 2 hours prior to imaging.

4. In a separate tube, dilute $1.5 \mu$ Lipofectamine 2000 in Opti-MEM to a total volume of $100 \mu$ l.

5. Add the $100 \mu$ l of diluted Lipofectamine 2000 (from Step 4) to the DNA solution (from Step 3) to create a 1:3 (wt:vol) ratio of DNA to Lipofectamine 2000.

6. Vortex the mixture for 10 seconds and incubate at room temperature for 10-15 minutes.

7. Refresh the culture medium of the HeLa cells with pre-warmed culture medium without antibiotics.

8. Add $50 \mu$ l of the transfection mixture per well and incubate the cells for 6 hours.

9. Refresh the culture medium of the transfected cells with $400 \mu \mathrm{l}$ pre-warmed phenol-red free full culture medium and incubate for another 18 hours.

(Dual-color) TIRF microscopy set up Timing 1.5 hours. 30 minutes hands on time.

10. Switch on the heating and $\mathrm{CO}_{2}$ control at least 1 hour in advance.

CRITICAL STEP We advise to use a microscope set-up equipped with a humidified stage incubator for imaging at $37{ }^{\circ} \mathrm{C}$ and $5 \% \mathrm{CO}_{2}$. However, in certain cells (e.g. HeLa and HUVEC) it is possible to detect MVB-PM fusion activity without stage incubator and/or imaging at room temperature $\left(21-24^{\circ} \mathrm{C}\right)$ in Tyrode's solution or another $\mathrm{CO}_{2}$-independent type of medium (e.g. Leibovitz's L15).

11. Start up the $488 \mathrm{~nm}$ laser line. In case of dual-color TIRF, also start up the $594 \mathrm{~nm}$ laser line.

12. Switch on microscope system and camera(s)

13. Start the imaging software (e.g. NIS-Elements)

14. Clean and dry the TIRF objective lens with $70 \%$ (vol/vol) ethanol. Adjust the correction collar of the objective for the right temperature and thickness of the glass coverslip. 
15. Focus and align the laser(s) so that the laser beam is focused on the back focal plane in the central axis of the objective.

16. Select the optical configuration for widefield GFP epi-fluorescence microscopy

17. Place the Lab-Tek chambered coverglass containing the transfected cells in the microscope stage holder.

18. Localize CD63-pHluorin expressing cells in widefield epi-fluorescence mode. ?Troubleshooting

19. Start the perfect focus system and focus on the basal plasma membrane.

20. Select the optical configuration for TIRF microscopy.

21. Set the TIRF angle by increasing the incidence angle of the excitation beam using the motorized TIRF illuminator unit until there is a sudden decrease in background signal from the interior of the cell and the plasma membrane has a two-dimensional appearance (Fig 4$)^{44}$.

CRITICAL STEP TIRF illumination is critical for the detection of MVB-PM fusion events with high sensitivity.

CRITICAL STEP In experiments where the number of TSPAN-pHluorin expressing cells is limited, setting of the TIRF angle can be done using $100 \mathrm{~nm}$ TetraSpeck Microspheres (Invitrogen, cat. no. T7279) as described in Ref ${ }^{44}$.

22. Optional: When performing dual-color TIRF imaging using two cameras, select the dualcamera mode and insert the TuCam filter cassette.

23. Optional: When performing dual-color TIRF imaging using two cameras, perform a horizontal and vertical alignment of the green and red channels in the center of the field of view.

CRITICAL STEP The two cameras must be aligned in z-depth to obtain the same focus for both channels.

CRITICAL STEP Potential remaining distortions can be corrected after image acquisition using image registration software.

CRITICAL STEP In experiments where the number of TSPAN-pHluorin expressing cells is limited, alignment of red and green channels can be done using $100 \mathrm{~nm}$ TetraSpeck Microspheres (Invitrogen, cat. no. T7279).

24. Adjust laser power and exposure time.

CRITICAL STEP Use the lowest laser intensity and shortest exposure time possible to avoid photobleaching and phototoxicity.

CRITICAL STEP Spectral crosstalk between the green and red channel will lead to false results. To control for this, excite with each laser separately and check if there is bleed-through of the fluorescent signal between channels.

25. Specify camera settings, we recommend to use a bit depth of 12 or 16 and no binning to obtain images of high resolution.

CRITICAL STEP The AMvBE macro criterium for 'minimum event size to be considered' (see Box 2) requires that the pixel size is small enough for fusion events to obey the Nyquist criterion (i.e. for the recommended 'minimum event size to be considered' value of $400 \mathrm{~nm}$, the pixel size should be a maximum of $200 \mathrm{~nm}$ ).

Image acquisition Timing in total 30-60 minutes per condition.

26. Choose an imaging field, ideally containing 2 or more cells with average expression levels. CRITICAL STEP It is critical to have a perfect focus at the basal plasma membrane. 
CRITICAL STEP For the analysis of MVB-PM fusion in dual-color, we recommend imaging cells that have similar expression levels of TSPAN-pHluorin and TSPAN-pHuji.

?Troubleshooting

27. Image the cells at $\geq 2 \mathrm{~Hz}$ for a time period that allows proper quantification (e.g. 3 minutes for HeLa cells).

CRITICAL STEP an imaging speed of $\geq 2 \mathrm{~Hz}$ is crucial to distinguish full MVB-PM fusion events from kiss-and-run-like events and neutral (transport) vesicles moving into the TIRF field.

?Troubleshooting

28. Repeat from steps $26-27$ for $\geq 5$ different fields.

Quantifying tetraspanin-pHluorin MVB-PM fusion events using the AMvBE macro Timing channel alignment of dual-color TIRF data: \pm 30 minutes per condition. Quantifying MVB-PM fusion events: \pm 30 minutes per condition.

CRITICAL Although manual analysis of fusion events based on the guidelines available in Box 1 is possible, we recommend using assisted counting using the AMvBE macro (Supplementary Software 1) for quantitative analysis of MVB-PM fusion events (Steps 30-37).

CRITICAL the AMvBE macro was developed to quantify MVB-PM fusion events using CD63-pHluorin. We do not recommend to use this tool with TSPAN-pHuji, due to the reduced $\mathrm{pH}$-sensitivity of this fluorescent protein.

29. Optional: When performing dual-color TIRF using two cameras, first correct small distortions in channel alignment using Image Registration in NIS-Elements. Load the acquisition data in NIS-Elements and select 'image' $\rightarrow$ 'Channel Alignment and Registration' $\rightarrow$ 'Image Registration'. Then select the channel you want to modify and select the 3-point (linear) transformation. Define three control points that can be used to align the two channels (e.g. retraction fibers, edges of the PM, filopodia). Select apply to all frames and click 'OK' to align the channels. Save the aligned acquisition data.

30. Install the plug-in: open FIJI and select 'Plugins' $\rightarrow$ 'Macros' $\rightarrow$ 'Install...' and open the AMvBE macro in the plugin folder.

CRITICAL STEP This step has to be repeated each time you restart FIJI.

CRITICAL STEP Ensure FIJI is updated to the latest version and is supplemented with the MultiStackReg and TurboReg plug-ins.

31. Load an image stack by selecting 'Plugins' $\rightarrow$ 'Macros' $\rightarrow$ 'AMvBE'. Navigate to the folder where the imaging data is saved and open the image.

32. When the image stack is loaded into FIJl, a browser appears. Select the location where you want the results of the image analysis to be stored.

33. Select the parameters for analysis in the window that appears. We recommend the following settings (at an acquisition framerate of $2 \mathrm{~Hz}$ ):

- Time window for event correction (in nb of frames): 6

- Condition for fluorescence decrease after exocytosis (min. $\mathrm{nb}$ of frames): 3

- Condition for fluorescence increase before exocytosis (max. nb of frames): 3

- Min. event size to be considered (in micrometer): 0.4

- If the decay condition is not fulfilled, threshold value $(N)$ for exocytosis event rescue such that Mean $+\mathrm{N}$ *STD: 3 
- Manual threshold for vesicle movement fixed by users (unit in nb of vesicle diameter): 2

CRITICAL STEP It is critical to enter the right settings for pixel size and framerate. Image registration to correct for cell movement is highly recommended to avoid that fusion events are discarded based on vesicle movement.

CRITICAL STEP Although photobleaching during image acquisition should be avoided, the AMvBE macro has the option to correct for bleaching.

34. Click ' $O K$ ' and the macro will start the image registration. After image registration, a dialogue box appears that says: 'Pinpoint the exocytosis event (left click) Right click to exit'. ?Troubleshooting

35. Click 'OK' and select all sudden increases in fluorescence intensity that appear fusion events. CRITICAL STEP fusion events within the first or last 3 seconds of the acquisition cannot be analyzed. Therefore, we recommend to exclude this time from further analysis.

36. After selecting all fusion events, right-click to open a dialogue box that says: 'Each event will be analyzed'.

37. Click 'OK' and the AMvBE macro will analyze all events. All results can be found in the destination folder that was selected in step 32.

\section{?Troubleshooting}

\section{Timing}

Step 2, seeding the cells: 15 minutes.

Step 3-8, transfection: \pm 30 minutes.

Step 9, refreshing culture medium: 5 minutes.

Step 10-22, (dual-color) TIRF microscopy set up: 1.5 hours, 30 minutes hands on time.

Step 26-28, live imaging of MVB-PM fusion, 30-60 minutes per condition

Step 29, channel alignment of dual-color TIRF data: \pm 30 minutes per condition.

Step 30-37, analysis of fusion activity using AMvBE: \pm 30 minutes per condition.

Box 1, manual analysis of fusion activity: 1 hour per condition.

\section{Troubleshooting}

Troubleshooting guidance can be found in Table 1.

Table 1. Troubleshooting Table

\begin{tabular}{|c|c|c|c|}
\hline step & Problem & Possible reason & Solution \\
\hline 18 & $\begin{array}{l}\text { Cells cannot be } \\
\text { localized. }\end{array}$ & $\begin{array}{l}\text { Transfection } \\
\text { efficiency is too } \\
\text { low. }\end{array}$ & $\begin{array}{l}\text { Increase the amount of } \\
\text { DNA:Lipofectamine } 2000 \\
\text { used for transfection or } \\
\text { vary DNA:Lipofectamine } \\
2000 \text { ratios from 1:0.5 } \\
\text { to } 1: 5 \text {. If this does not } \\
\text { increase the } \\
\text { transfection efficiency, } \\
\text { or results in cell toxicity, } \\
\text { use another transfection }\end{array}$ \\
\hline
\end{tabular}




\begin{tabular}{|c|c|c|c|}
\hline & & & $\begin{array}{l}\text { reagent or } \\
\text { electroporation } \\
\text { procedure (e.g. Neon } \\
\text { system, Thermofischer). } \\
\text { Alternatively, lentiviral } \\
\text { transduction can be } \\
\text { used for difficult-to- } \\
\text { transfect cells. }\end{array}$ \\
\hline \multirow[t]{3}{*}{$26-27$} & $\begin{array}{l}\text { Many visible (pH } \\
\text { neutral) } \mathrm{CD} 63- \\
\text { pHluorin } \\
\text { positive } \\
\text { compartments } \\
\text { in field of view. }\end{array}$ & $\begin{array}{l}\text { Microscope is not } \\
\text { in TIRF mode. }\end{array}$ & $\begin{array}{l}\text { Reset the TIRF angle } \\
\text { (Step 22) and focus on } \\
\text { the plasma membrane } \\
\text { (Fig. 3). }\end{array}$ \\
\hline & & $\begin{array}{l}\text { CD63-pHluorin is } \\
\text { trapped in the } \\
\text { endoplasmic } \\
\text { reticulum. }\end{array}$ & $\begin{array}{l}\text { Image cells with lower } \\
\text { CD63-pHluorin } \\
\text { expression or transfect } \\
\text { cells with less CD63- } \\
\text { pHluorin DNA. }\end{array}$ \\
\hline & & $\begin{array}{l}\text { Cells contain } \\
\text { endosomes with a } \\
\text { (close to) neutral } \\
\text { pH. }\end{array}$ & $\begin{array}{l}\text { Although the presence } \\
\text { of these visible } \\
\text { endosomes makes the } \\
\text { detection of MVB-PM } \\
\text { fusion events more } \\
\text { challenging, fusion } \\
\text { events can still be } \\
\text { detected. }\end{array}$ \\
\hline \multirow[t]{3}{*}{27} & $\begin{array}{l}\text { No fusion events } \\
\text { can be detected. }\end{array}$ & $\begin{array}{l}\text { The cell type that } \\
\text { is studied has no } \\
\text { or very low fusion } \\
\text { activity. }\end{array}$ & $\begin{array}{l}\text { Use HeLa or HEK } 293 \\
\text { cells as positive control } \\
\text { to make sure that the } \\
\text { imaging set-up is } \\
\text { correct. }\end{array}$ \\
\hline & & $\begin{array}{l}\text { The cells do not } \\
\text { contain many } \\
\text { acidic CD63- } \\
\text { pHluorin positive } \\
\text { compartments. }\end{array}$ & $\begin{array}{l}\text { Use } \mathrm{NH}_{4} \mathrm{Cl} \text { wash ( } 50 \mathrm{mM} \\
\mathrm{NH}_{4} \mathrm{Cl} \text { in Tyrode's } \\
\text { solution, } \mathrm{pH} 7.4 \text { ) to } \\
\text { visualize } \mathrm{CD} 63-\mathrm{pH} \text { luorin } \\
\text { positive acidic MVBs. }\end{array}$ \\
\hline & & $\begin{array}{l}\text { The microscope is } \\
\text { not focused at the } \\
\text { cellular plasma } \\
\text { membrane. }\end{array}$ & $\begin{array}{l}\text { Use acid wash (Tyrode's } \\
\text { solution, pH 5.5) to } \\
\text { quench CD63-pHluorin } \\
\text { on the PM. If the } \\
\text { fluorescent signal does } \\
\text { not decrease this means } \\
\text { the focus is not at the }\end{array}$ \\
\hline
\end{tabular}




\begin{tabular}{|c|c|c|c|}
\hline & & & $\begin{array}{l}\text { plasma membrane and } \\
\text { the imaging is not } \\
\text { performed in TIRF. } \\
\text { Reset the TIRF angle } \\
\text { (Step 22) and focus on } \\
\text { the plasma membrane } \\
\text { (Fig. 3). }\end{array}$ \\
\hline \multirow[t]{2}{*}{27} & $\begin{array}{l}\text { Cells become } \\
\text { smaller over } \\
\text { time. }\end{array}$ & $\begin{array}{l}\text { High laser } \\
\text { intensity causes } \\
\text { phototoxicity. }\end{array}$ & $\begin{array}{l}\text { Reduce laser power or } \\
\text { acquisition time. }\end{array}$ \\
\hline & & $\begin{array}{l}\mathrm{CO}_{2} \text { control is not } \\
\text { on, resulting in } \\
\text { increased } \\
\text { phototoxicity. }\end{array}$ & $\begin{array}{l}\text { Turn on } \mathrm{CO}_{2} \text { control or } \\
\text { use } \mathrm{CO}_{2} \text {-independent } \\
\text { medium }\end{array}$ \\
\hline 27 & $\begin{array}{l}\text { Fluorescence } \\
\text { intensity } \\
\text { decreases over } \\
\text { time. }\end{array}$ & $\begin{array}{l}\text { High laser } \\
\text { intensity induces } \\
\text { photobleaching of } \\
\text { CD63-pHluorin. }\end{array}$ & $\begin{array}{l}\text { Reduce laser power or } \\
\text { acquisition time }\end{array}$ \\
\hline 34 & $\begin{array}{l}\text { A pop-up } \\
\text { window appears } \\
\text { stating that } \\
\text { TurboReg or } \\
\text { MultiStackReg is } \\
\text { not (properly) } \\
\text { installed. }\end{array}$ & $\begin{array}{l}\text { TurboReg and/or } \\
\text { MultiStackReg } \\
\text { is/are not } \\
\text { (properly) } \\
\text { installed. }\end{array}$ & $\begin{array}{l}\text { Reinstall TurboReg } \\
\text { and/or MultiStackReg } \\
\text { according to the } \\
\text { description in the } \\
\text { Readme file. After } \\
\text { installing TurboReg } \\
\text { and/or MultiStackReg, } \\
\text { restart FIJ software and } \\
\text { reinstall AMvBE. } \\
\text { Alternatively, stacks can } \\
\text { be preregistered and } \\
\text { then analysed with } \\
\text { AMvBE without } \\
\text { registration by } \\
\text { unchecking the } \\
\text { "registration to correct } \\
\text { cell movement" box. }\end{array}$ \\
\hline 37 & $\begin{array}{l}\text { Unexpected } \\
\text { values in } \\
\text { summary .csv } \\
\text { files when } \\
\text { opened in Excel. }\end{array}$ & $\begin{array}{l}\text { Decimal and } \\
\text { Thousand } \\
\text { separator settings } \\
\text { in Excel are } \\
\text { incorrect. }\end{array}$ & $\begin{array}{l}\text { Change Decimal and } \\
\text { Thousands separator } \\
\text { settings for all columns. } \\
\text { Decimal separator: (,) } \\
\text { and Thousands } \\
\text { separator: () (space). }\end{array}$ \\
\hline
\end{tabular}

\section{Anticipated results}


This protocol describes the live imaging of MVB-PM fusion using TSPAN-reporters on a TIRF setup. Successful implementation of the protocol provides insight into the quantity and (subcellular)localization of exosome release at single-cell resolution. This approach can be used to investigate exosome release in a wide variety of adherent cell types. So far, we have successfully used the CD63-pHluorin reporter in HeLa, HUVEC, HEK293T, Caski, SiHa, HCT116, Caco-2, MSC and MNT-1 cells. Supplementary Videos 1, 6 and 7 show typical examples of CD63-pHluorin MVB-PM fusion events as observed with live cell TIRF microscopy in HeLa, HUVEC and HEK293T cells, respectively. Average fusion activity varies between different cell lines, and there is substantial variation in fusion activity between cells, even within the same experiment (Fig. 5a).

The TSPAN-reporter live cell imaging approach can also be used to directly assess the influence of external stimuli on MVB-PM fusion activity, as shown here with the small molecule histamine (Fig. 5b). Due to the high variation in basal fusion activity between cells, even within one experiment, stimuli that cause subtle modulations of the fusion activity are likely to be missed in a pooled, unpaired, analysis. Therefore, we recommend to measure fusion activity in single cells at baseline, and during (or after) the stimulus. Data can then be visualized by plotting fusion activity (the number of MVB-PM fusion events per minute) of each individual cell at baseline and in the presence of the stimulus (Fig. $5 b)$. To present fusion activity data in a more visual way, we routinely use a total projection of all fusion events over a given time period on the background of the cell (Fig. 5c), which enables visualization of the location of MVB-PM fusion events in single cells, and might reveal hotspots of exosome release. We did not observe preferential sites for MVB exocytosis in HeLa, HUVEC or HEK239T cells, which might be explained by the limited migration of these cell lines.

Compared to manual analysis, the AMvBE macro provides a faster and more objective way of analyzing basal MVB-PM fusion activity (Fig. 5a) or modulation of MVB-PM fusion activity (Fig. 5b). Furthermore, analysis with the AMvBE macro generates multiple output files that can be used to control and display the results. For example, AMvBE creates a summary TIFF file of the acquisition where the pinpointed fusion events are highlighted with colored circles depending on the outcome of the analysis (Fig. $5 \mathrm{~d}$; Supplementary Video 8). In addition, intensity profiles are automatically saved for the true MVB-PM fusion events, which can be used to visualize and assess decay kinetics of individual events (Fig. 3a). Alternatively, individual fusion events can be visualized in a heatmap time-lapse gallery (Fig. 3b). The list with frame numbers containing true MVB-PM fusion events can be used to make a total projection of all MVB exocytosis events over a time-course of several minutes (Fig. 5c).

Simultaneous dual-color TIRF microscopy of a combination of TSPAN-pHluorins with red fluorescent proteins can be used to track MVBs before fusion or to identify cargo molecules of exocytic MVBs. This data can be represented best by a time-lapse gallery (Fig. 2a) or a fluorescent intensity profile of the fusion event in both channels. We observed multiple double-positive MVB-PM fusion events in HeLa cells expressing CD63-pHluorin and CD81-pHuji (Fig. 2a; Supplementary Video 2).

\section{Reporting Summary}

Further information on research design is available in the Nature Research Reporting Summary linked to this article.

\section{Data availability}


The data that support this study are available from the corresponding author upon reasonable request.

\section{Code availability}

The AMvBE macro can be found in Supplementary Software 1

\section{Author contributions statements}

M.P.B. and F.J.V. performed the experiments, prepared the figures and wrote the manuscript. G.v.N, P.B., S.H. and D.M.P. provided critical feedback and helped shape the manuscript. P.B. developed the AMvBE macro. S.H. helped setting up the dual-color TIRF experiments. D.M.P. conceived the study. F.J.V. and D.M.P. supervised the study.

\section{Acknowledgments}

We acknowledge Neurlmag facility of the Institute of Psychiatry and Neuroscience of Paris where the AMvBE macro has been developed. This protocol was funded by a Dutch Organizations for Scientific Research-Amsterdam Institute for Molecules, Medicines and Systems STAR Graduate Program grant (022.005.031) to M.P.B., a Dutch Cancer Fund (KWF-5510) and a Cancer Center Amsterdam-VU University Medical Center grant to D.M.P., and a European Molecular Biology Organization grant (EMBO ALTF 1383-2014) and a Fondation ARC pour la Recherché sur le Cancer fellowship (PJA 20161204808) to F.J.V.

\section{Competing interests}

The authors declare that they have no competing financial interests.

\section{References}

1 Huotari, J. \& Helenius, A. Endosome maturation. EMBO J 30, 3481-3500, doi:10.1038/emboj.2011.286 (2011).

2 van Niel, G., D'Angelo, G. \& Raposo, G. Shedding light on the cell biology of extracellular vesicles. Nat Rev Mol Cell Biol, doi:10.1038/nrm.2017.125 (2018).

3 Bebelman, M. P., Smit, M. J., Pegtel, D. M. \& Baglio, S. R. Biogenesis and function of extracellular vesicles in cancer. Pharmacol Ther, doi:10.1016/j.pharmthera.2018.02.013 (2018).

4 Costa-Silva, B. et al. Pancreatic cancer exosomes initiate pre-metastatic niche formation in the liver. Nat Cell Biol 17, 816-826, doi:10.1038/ncb3169 (2015).

5 Peinado, H. et al. Melanoma exosomes educate bone marrow progenitor cells toward a prometastatic phenotype through MET. Nat Med 18, 883-891, doi:10.1038/nm.2753 (2012).

6 Bobrie, A., Colombo, M., Raposo, G. \& Thery, C. Exosome secretion: molecular mechanisms and roles in immune responses. Traffic 12, 1659-1668, doi:10.1111/j.1600-0854.2011.01225.x (2011).

7 Bian, S. et al. Extracellular vesicles derived from human bone marrow mesenchymal stem cells promote angiogenesis in a rat myocardial infarction model. J Mol Med (Berl) 92, 387-397, doi:10.1007/s00109-013-1110-5 (2014).

8 Tkach, M. \& Thery, C. Communication by Extracellular Vesicles: Where We Are and Where We Need to Go. Cell 164, 1226-1232, doi:10.1016/j.cell.2016.01.043 (2016).

$9 \quad$ Kowal, J. et al. Proteomic comparison defines novel markers to characterize heterogeneous populations of extracellular vesicle subtypes. Proc Natl Acad Sci U S A 113, E968-977, doi:10.1073/pnas.1521230113 (2016). 
10 van der Vlist, E. J., Nolte-'t Hoen, E. N., Stoorvogel, W., Arkesteijn, G. J. \& Wauben, M. H. Fluorescent labeling of nano-sized vesicles released by cells and subsequent quantitative and qualitative analysis by high-resolution flow cytometry. Nat Protoc 7, 1311-1326, doi:10.1038/nprot.2012.065 (2012).

11 Zhang, H. \& Lyden, D. Asymmetric-flow field-flow fractionation technology for exomere and small extracellular vesicle separation and characterization. Nat Protoc 14, 1027-1053, doi:10.1038/s41596-019-0126-x (2019).

12 Gardiner, C. et al. Techniques used for the isolation and characterization of extracellular vesicles: results of a worldwide survey. J Extracell Vesicles 5, 32945, doi:10.3402/jev.v5.32945 (2016).

13 Pegtel, D. M. \& Gould, S. J. Exosomes. Annu Rev Biochem 88, 487-514, doi:10.1146/annurevbiochem-013118-111902 (2019).

14 Miesenbock, G., De Angelis, D. A. \& Rothman, J. E. Visualizing secretion and synaptic transmission with pH-sensitive green fluorescent proteins. Nature 394, 192-195, doi:10.1038/28190 (1998).

15 Sankaranarayanan, S., De Angelis, D., Rothman, J. E. \& Ryan, T. A. The use of pHluorins for optical measurements of presynaptic activity. Biophys J 79, 2199-2208, doi:10.1016/S00063495(00)76468-X (2000).

16 Sung, B. H., Ketova, T., Hoshino, D., Zijlstra, A. \& Weaver, A. M. Directional cell movement through tissues is controlled by exosome secretion. Nat Commun 6, 7164, doi:10.1038/ncomms 8164 (2015).

17 Verweij, F. J. et al. Quantifying exosome secretion from single cells reveals a modulatory role for GPCR signaling. J Cell Biol, doi:10.1083/jcb.201703206 (2018).

18 Lu, A. et al. Genome-wide interrogation of extracellular vesicle biology using barcoded miRNAs. Elife 7, doi:10.7554/eLife.41460 (2018).

19 Gutknecht, J. Proton/hydroxide conductance and permeability through phospholipid bilayer membranes. Proc Natl Acad Sci U S A 84, 6443-6446, doi:10.1073/pnas.84.18.6443 (1987).

20 Paula, S., Volkov, A. G., Van Hoek, A. N., Haines, T. H. \& Deamer, D. W. Permeation of protons, potassium ions, and small polar molecules through phospholipid bilayers as a function of membrane thickness. Biophys J 70, 339-348, doi:10.1016/S0006-3495(96)79575-9 (1996).

21 Raven, J. A. \& Beardall, J. The intrinsic permeability of biological membranes to $\mathrm{H}+$ : Significance for the efficiency of low rates of energy transformation. FEMS Microbiology Letters 10, 1-5, doi:10.1111/j.1574-6968.1981.tb06194.x (1981).

22 Verweij, F. J. et al. LMP1 association with CD63 in endosomes and secretion via exosomes limits constitutive NF-kappaB activation. EMBO J 30, 2115-2129, doi:10.1038/emboj.2011.123 (2011).

23 Sung, B. H., Pelletier, R. \& Weaver, A. M. pHluo_M153R-CD63, a bright, versatile live cell reporter of exosome secretion and uptake, reveals pathfinding behavior of migrating cells. bioRxiv, 577346, doi:10.1101/577346 (2019).

24 Mittelbrunn, M. et al. Unidirectional transfer of microRNA-loaded exosomes from T cells to antigen-presenting cells. Nat Commun 2, 282, doi:10.1038/ncomms1285 (2011).

25 van Niel, G. et al. Intestinal epithelial cells secrete exosome-like vesicles. Gastroenterology 121, 337-349 (2001).

26 Hoshino, D. et al. Exosome secretion is enhanced by invadopodia and drives invasive behavior. Cell Rep 5, 1159-1168, doi:10.1016/j.celrep.2013.10.050 (2013).

27 Verweij, F. J. et al. Live Tracking of Inter-organ Communication by Endogenous Exosomes In Vivo. Dev Cell 48, 573-589 e574, doi:10.1016/j.devcel.2019.01.004 (2019).

28 Van Deun, J. et al. EV-TRACK: transparent reporting and centralizing knowledge in extracellular vesicle research. Nat Methods 14, 228-232, doi:10.1038/nmeth.4185 (2017). 
29 van der Pol, E. et al. Particle size distribution of exosomes and microvesicles determined by transmission electron microscopy, flow cytometry, nanoparticle tracking analysis, and resistive pulse sensing. J Thromb Haemost 12, 1182-1192, doi:10.1111/jth.12602 (2014).

30 Yuana, Y., Levels, J., Grootemaat, A., Sturk, A. \& Nieuwland, R. Co-isolation of extracellular vesicles and high-density lipoproteins using density gradient ultracentrifugation. J Extracell Vesicles 3, doi:10.3402/jev.v3.23262 (2014).

31 Coumans, F. A. W. et al. Methodological Guidelines to Study Extracellular Vesicles. Circ Res 120, 1632-1648, doi:10.1161/CIRCRESAHA.117.309417 (2017).

32 Linares, R., Tan, S., Gounou, C., Arraud, N. \& Brisson, A. R. High-speed centrifugation induces aggregation of extracellular vesicles. J Extracell Vesicles 4, 29509, doi:10.3402/jev.v4.29509 (2015).

33 Jeppesen, D. K. et al. Reassessment of Exosome Composition. Cell 177, 428-445 e418, doi:10.1016/j.cell.2019.02.029 (2019).

34 Zhang, H. et al. Identification of distinct nanoparticles and subsets of extracellular vesicles by asymmetric flow field-flow fractionation. Nat Cell Biol 20, 332-343, doi:10.1038/s41556-018-0040-4 (2018).

35 Messenger, S. W., Woo, S. S., Sun, Z. \& Martin, T. F. J. A Ca(2+)-stimulated exosome release pathway in cancer cells is regulated by Munc13-4. J Cell Biol 217, 2877-2890, doi:10.1083/jcb.201710132 (2018).

36 Nabhan, J. F., Hu, R., Oh, R. S., Cohen, S. N. \& Lu, Q. Formation and release of arrestin domain-containing protein 1-mediated microvesicles (ARMMs) at plasma membrane by recruitment of TSG101 protein. Proc Natl Acad Sci U S A 109, 4146-4151, doi:10.1073/pnas.1200448109 (2012). 37 Colombo, M., Raposo, G. \& Thery, C. Biogenesis, secretion, and intercellular interactions of exosomes and other extracellular vesicles. Annu Rev Cell Dev Biol 30, 255-289, doi:10.1146/annurevcellbio-101512-122326 (2014).

38 Shen, Y., Rosendale, M., Campbell, R. E. \& Perrais, D. pHuji, a pH-sensitive red fluorescent protein for imaging of exo- and endocytosis. J Cell Biol 207, 419-432, doi:10.1083/jcb.201404107 (2014).

39 de Wit, J., Toonen, R. F. \& Verhage, M. Matrix-dependent local retention of secretory vesicle cargo in cortical neurons. J Neurosci 29, 23-37, doi:10.1523/JNEUROSCI.3931-08.2009 (2009).

40 Urbina, F. L., Gomez, S. M. \& Gupton, S. L. Spatiotemporal organization of exocytosis emerges during neuronal shape change. J Cell Biol 217, 1113-1128, doi:10.1083/jcb.201709064 (2018).

41 Yuan, T., Lu, J., Zhang, J., Zhang, Y. \& Chen, L. Spatiotemporal detection and analysis of exocytosis reveal fusion "hotspots" organized by the cytoskeleton in endocrine cells. Biophys $J \mathbf{1 0 8}$, 251-260, doi:10.1016/j.bpj.2014.11.3462 (2015).

42 Schindelin, J. et al. Fiji: an open-source platform for biological-image analysis. Nat Methods 9, 676-682, doi:10.1038/nmeth.2019 (2012).

43 Thevenaz, P., Ruttimann, U. E. \& Unser, M. A pyramid approach to subpixel registration based on intensity. IEEE Trans Image Process 7, 27-41, doi:10.1109/83.650848 (1998).

44 Mattheyses, A. L., Simon, S. M. \& Rappoport, J. Z. Imaging with total internal reflection fluorescence microscopy for the cell biologist. J Cell Sci 123, 3621-3628, doi:10.1242/jcs.056218 (2010).

\section{Figure legends main text}

\section{Fig. 1: Model for the visualization of MVB-PM fusion with CD63-pHluorin}

A pH-sensitive optical reporter (CD63-pHluorin) is quenched when facing the acidic lumen of the MVB. Upon fusion of the MVB with the PM, low luminal $\mathrm{pH}$ is immediately neutralized, resulting in a sudden increase in fluorescent intensity that can be detected by live microscopy. Insert upper right: Stills from 
a CD63-pHluorin MVB-PM fusion event as visualized by live Total Internal Reflection Fluorescence (TIRF) microscopy $1 \mathrm{~s}$ before fusion (1), at the moment of fusion (2), and $30 \mathrm{~s}$ after fusion (3). Scale bar, $2.5 \mu \mathrm{m}$. EC, extracellular. (modified from ${ }^{17}$ ).

Fig. 2: Simultaneous dual-color TIRF microscopy of TSPAN-pHluorin with TSPAN-pHuji reporters

a, (Left) Merged stills of dual-color TIRF imaging with CD63-pHluorin and CD81-pHuji (upper), CD63pHluorin and CD63-mRFP (middle) and CD63-C-term-pHluorin and CD63-pHuji (lower, original source ${ }^{17}$ ) at the moment of MVB-PM fusion (in the white dashed square). Scale bars, $20 \mu \mathrm{m}$. (Right) Enlarged stills of the MVB-PM fusion events 1 second before fusion, at the moment of fusion, and 10 seconds after fusion. $\mathbf{b}$, Models (upper) and theoretical intensity plots (lower) of MVB-PM fusion events for the different combinations of TSPAN-reporters. c, Comparison of peak fluorescence intensities (as fold over background) of MVB-PM fusion events in the green and red channel of CD63-pHluorin and CD63pHuji co-expressing cells (63 individual fusion events in $n=20$ different cells). Black dashed line represents linear regression of all MVB-PM fusion events (Pearson's $r=0.78$, slope $=0.61$ ).

\section{Fig. 3: Visualization of individual fusion events}

a, Example of the fluorescence intensity profiles of a full MVB-PM fusion event, a kiss-and-run-like fusion event and a neutral vesicle moving in and out of the TIRF field in HeLa cells over time. $\mathbf{b}$, Heatmap time-lapse galleries of different types of CD63-pHluorin events obtained in HeLa cells. Full MVB-PM fusion and exosome release is characterized by a sudden increase in fluorescent intensity, followed by an exponential decrease of fluorescence at a fixed position. Kiss-and-run-like fusion events display a sudden increase in fluorescent intensity, but can be distinguished based on postfusion movement and irregular decrease of fluorescent signal. Neutral vesicles can be observed during their approach to the PM, resulting in a slower, more irregular increase in fluorescent intensity and can move in the TIRF field for prolonged time-periods. Note the difference in time scale between the kiss-and-run-like and the neutral vesicle time-lapses. c, Schematic explaining the various parameters of the AMvBE macro. (1) time point of fusion as pinpointed by user, (2) time window for event correction in which AMvBE determines the time point of the fluorescence intensity peak, (3) fluorescence intensity peak, (4) background fluorescence intensity, (5) maximum intensity of the fusion event, (6) time point during fluorescence increase where $I(t)=I_{\max } * e^{\left(\frac{t=\tau}{\tau}\right)}$ with $\tau=$ increase rate, (7) duration of the fluorescence increase, (8) time point during fluorescence decrease where $I(t)=I_{\max } * e^{-\left(\frac{t=\tau}{\tau}\right)}$ with $\tau=$ decay rate, (9) duration of fluorescence decrease, $(10)$ vesicle diameter (full width at half the maximum intensity of the fusion event), (11) maximum total movement of the fusion event (two times the vesicle diameter from the place of maximum intensity of the fusion event).

\section{Fig. 4: Setting the TIRF angle for a CD63-pHluorin expressing HeLa cell}

A CD63-pHluorin expressing HeLa cell imaged using epi-fluorescence microscopy at a $0^{\circ}$ angle (left) and at the correct TIRF angle (right). Scale bar, $20 \mu \mathrm{m}$.

\section{Fig. 5: Analysis of MVB-PM fusion activity with the AMvBE macro}

a, Basal MVB-PM fusion activity (fusion events per minute) measured in HeLa ( $n=25$ cells), HUVEC ( $n$ $=12$ cells) and HEK293T ( $n=12$ cells) and analyzed using the AMvBE macro. Data is represented as box-and-whisker plot where the line in the box represents the median, the boundaries of the box represent the $25^{\text {th }}$ and $75^{\text {th }}$ percentiles and the whiskers show the minimum and maximum values. *, 
$\mathrm{P}<0.05 ; * *, \mathrm{P}<0.01, * * * *, \mathrm{P}<0.0001$ using an unpaired Student's two-tailed two-sample $\mathrm{t}$ test. HeLa vs HUVEC: $P<0.0001$, HeLa vs HEK293T $P=0.0177$, HEK293T vs HUVEC: $P=0.0035$. b, Measurement of MVB-PM fusion activity in individual HeLa cells $(n=25)$ before and during stimulation with histamine $(100 \mu \mathrm{M})$ as analyzed with the AMvBE macro. Fusion activity before histamine stimulation is the same as basal fusion activity in HeLa in Fig. 5a. ${ }^{*}, \mathrm{P}<0.05$ using a paired Student's two-tailed two-sample t test. Ctrl vs $100 \mu \mathrm{M}$ histamine $\mathrm{P}=0.0386$. c, Projection of MVB-PM fusion events (yellow spots) in a HeLa cell (blue) over a time-course of 3 minutes. Scale bar, $20 \mu \mathrm{m}$. d, (Upper) Still of summary results file created by the AMvBE macro of a CD63-pHluorin expressing HeLa cell. Dashed white square depicts region of interest used for Supplementary Video 8. Scale bar, $20 \mu \mathrm{m}$. (Lower) Stills from Supplementary Video 8 showing a neutral CD63-pHluorin-positive vesicle moving in the TIRF plane (left; highlighted by a red ring) and a true MVB-PM fusion event (right; highlighted by a green ring). Scale bars, $5 \mu \mathrm{m}$.

\section{Supplementary Information}

Supplementary Video 1: TIRF microscopy of a CD63-pHluorin expressing HeLa cell at 8x speed. Scale bar $20 \mu \mathrm{m}$.

Supplementary Video 2: Dual-color TIRF microscopy of a CD63-pHluorin (green) and CD81-pHuji (red) expressing HeLa cell at $8 x$ speed. Scale bar $20 \mu \mathrm{m}$.

Supplementary Video 3: Dual-color TIRF microscopy of a CD63-pHluorin (green) and CD63-mRFP (red) expressing HeLa cell at 8x speed. Scale bar $20 \mu \mathrm{m}$. White arrows highlight MVBs containing CD63-pHluorin and CD63-mRFP that are visible in red before fusion.

Supplementary Video 4: Dual-color TIRF microscopy of a CD63-C-term-pHluorin (green) and CD63pHuji (red) expressing HeLa cell at $8 x$ speed. Scale bar $20 \mu \mathrm{m}$. Original source ${ }^{17}$.

Supplementary Video 5: TIRF microscopy of HeLa cells stably expressing CD63-pHluorin 6 weeks post-transduction at $8 \mathrm{x}$ speed. Scale bar $20 \mu \mathrm{m}$.

Supplementary Video 6: TIRF microscopy of a CD63-pHluorin expressing HUVEC cell at 8x speed. Scale bar $20 \mu \mathrm{m}$.

Supplementary Video 7: TIRF microscopy of a CD63-pHluorin expressing HEK293T cell at 8x speed. Scale bar $20 \mu \mathrm{m}$.

Supplementary Video 8: Example of the summary TIFF file as created by the AMvBE macro upon analysis of an MVB-PM fusion event (highlighted by a green circle) or a neutral CD63-pHluorinpositive vesicle moving in the TIRF plane (highlighted by a red circle). Scale bar, $5 \mu \mathrm{m}$.

Supplementary Software 1: AMvBE macro. 
Box 1 Criteria for manual counting of MVB-PM fusion events.

A burst of fluorescence is typically considered as exosome release/an MVB-PM fusion event when:

- There is a sudden increase in fluorescence intensity followed by an exponential decrease of the fluorescent signal.

- It reaches peak intensity in a maximum of 3 frames (when imaging at $2 \mathrm{~Hz}$ ).

- It has a circular shape with a diameter of at least $400 \mathrm{~nm}$.

- It shows minimal movement after the initial burst. Vesicles that move more than twice their diameter are excluded.

- It has a signal duration of $\geq 2 \mathrm{sec}$. Signal duration is defined as the time from peak intensity to the moment that the fluorescence signal at the fusion site is equal to the surrounding background fluorescence

Box 2 Description and recommended parameter settings for the analysis of MVB-PM fusion events with AMvBE.

The following settings are recommended for the analysis of MVB-PM fusion activity with AMvBE:

\section{- Time window for event correction (in nb of frames): 6}

During the selection of the events, the user might pinpoint a fusion event some frames before or after the peak fluorescence intensity of the event is reached. The time window for event correction allows $A M v B E$ to determine the maximum fluorescence intensity of the event 6 frames before and after the frame pinpointed by the user (Fig 3c, 1,2). The higher this value, the less accurate the user has to pinpoint the right frame. However, higher values might result in problems when there are multiple fusion events that occur close together in space and time.

- Condition for fluorescence decrease after exocytosis (min. nb of frames): 3

The fluorescence decay after fusion is fitted as exponential decay with $I(t)=$ $I_{\text {max }} * e^{-\left(\frac{t}{\tau}\right)}$, where $\mathrm{I}=$ intensity and $\tau$ is the decay rate. The duration of the fluorescence decay is the time from peak fluorescence intensity until the timepoint where $I(t)=I_{\max } * e^{-\left(\frac{t=\tau}{\tau}\right)}$, which corresponds to the timepoint where the intensity is \pm 0.37 times the maximum intensity (Fig 3c, 3-5,8,9).

- Condition for fluorescence increase before exocytosis (max. $n b$ of frames): 3

The fluorescence increase before fusion is fitted as exponential growth with $I(t)=$ $I_{\max } * e^{\left(\frac{t}{\tau}\right)}$, where I $=$ intensity and $\tau$ is the increase rate. The duration of the fluorescence increase is the time from the timepoint where $I(t)=I_{\max } * e^{\left(\frac{t=\tau}{\tau}\right)}$ until the peak fluorescent intensity is reached (Fig 3c, 3-7).

- Min. event size to be considered (in micrometer): 0.4

The size of the fusion event (vesicle diameter) is measured as the full width at half the maximum intensity of the fusion event (Fig 3c, 10). 


\begin{tabular}{|c|c|}
\hline - & $\begin{array}{l}\text { If the decay condition is not fulfilled, threshold value }(\mathbf{N}) \text { for exocytosis event } \\
\text { rescue such that Mean }+\mathbf{N} \text { *STD: } \mathbf{3} \\
\text { The fusion of MVBs that contain a relatively high TSPAN-pHluorin concentration in } \\
\text { the limiting membrane can result in a short fluorescence decrease time until } \\
I(t)=I_{\text {max }} * e^{-\left(\frac{t=\tau}{\tau}\right)} \text { is reached, followed by a slower residual decrease of the } \\
\text { remaining exosome-associated TSPAN-pHluorin fluorescence. Optionally, AMvBE } \\
\text { can rescue these MVB-PM fusion events by setting this parameter at 3. If deemed } \\
\text { inappropriate, this parameter can be set at } 100 \text { to avoid rescue of these events. } \\
\text { Manual threshold for vesicle movement fixed by users (unit in nb of vesicle } \\
\text { diameter): } \mathbf{2} \\
\text { To avoid counting kiss-and-run-like fusion events, the maximum movement of the } \\
\text { fluorescent signal after fusion is restricted to twice the vesicle diameter (Fig } 3 c \text {, } \\
\text { 10,11) }\end{array}$ \\
\hline
\end{tabular}




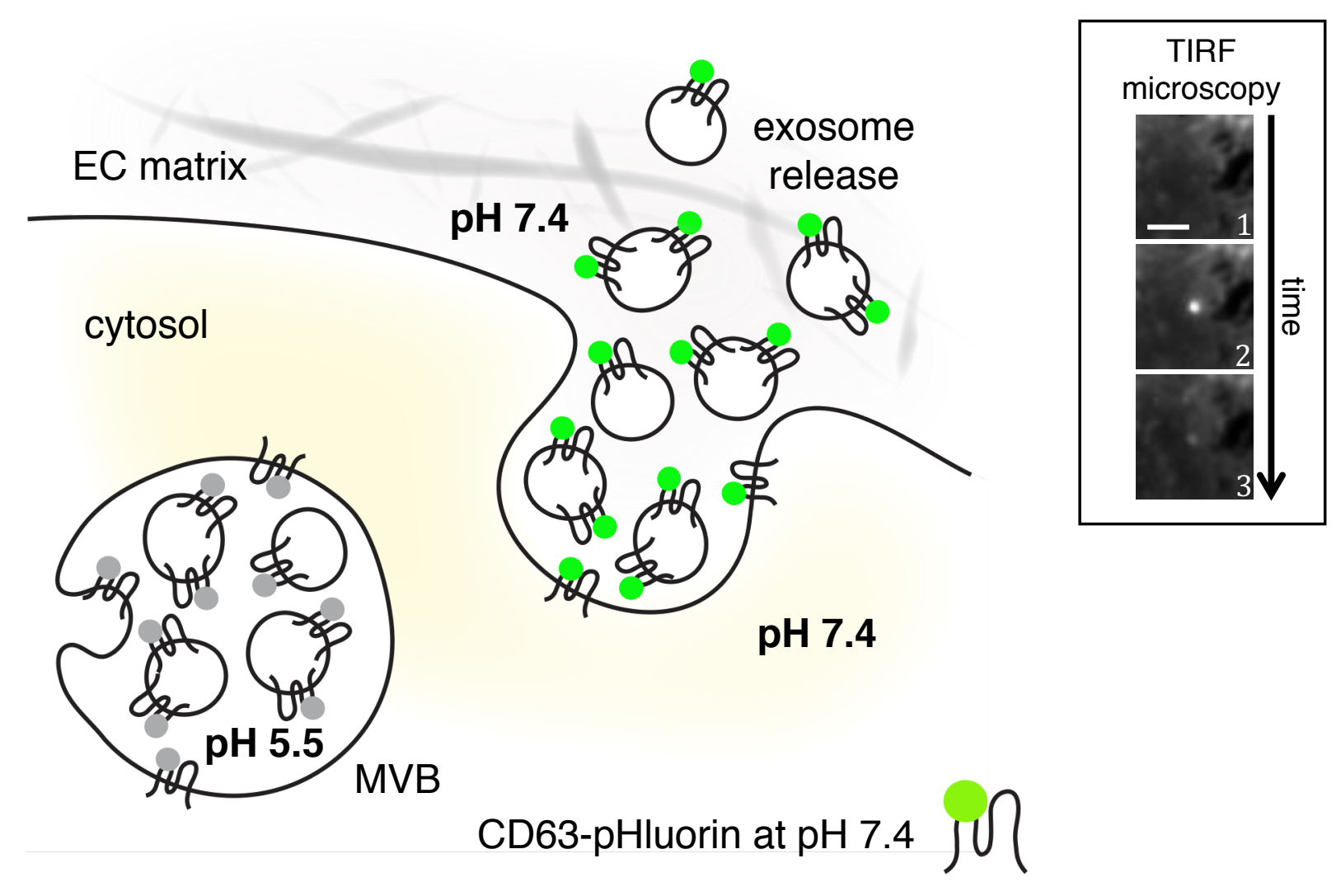




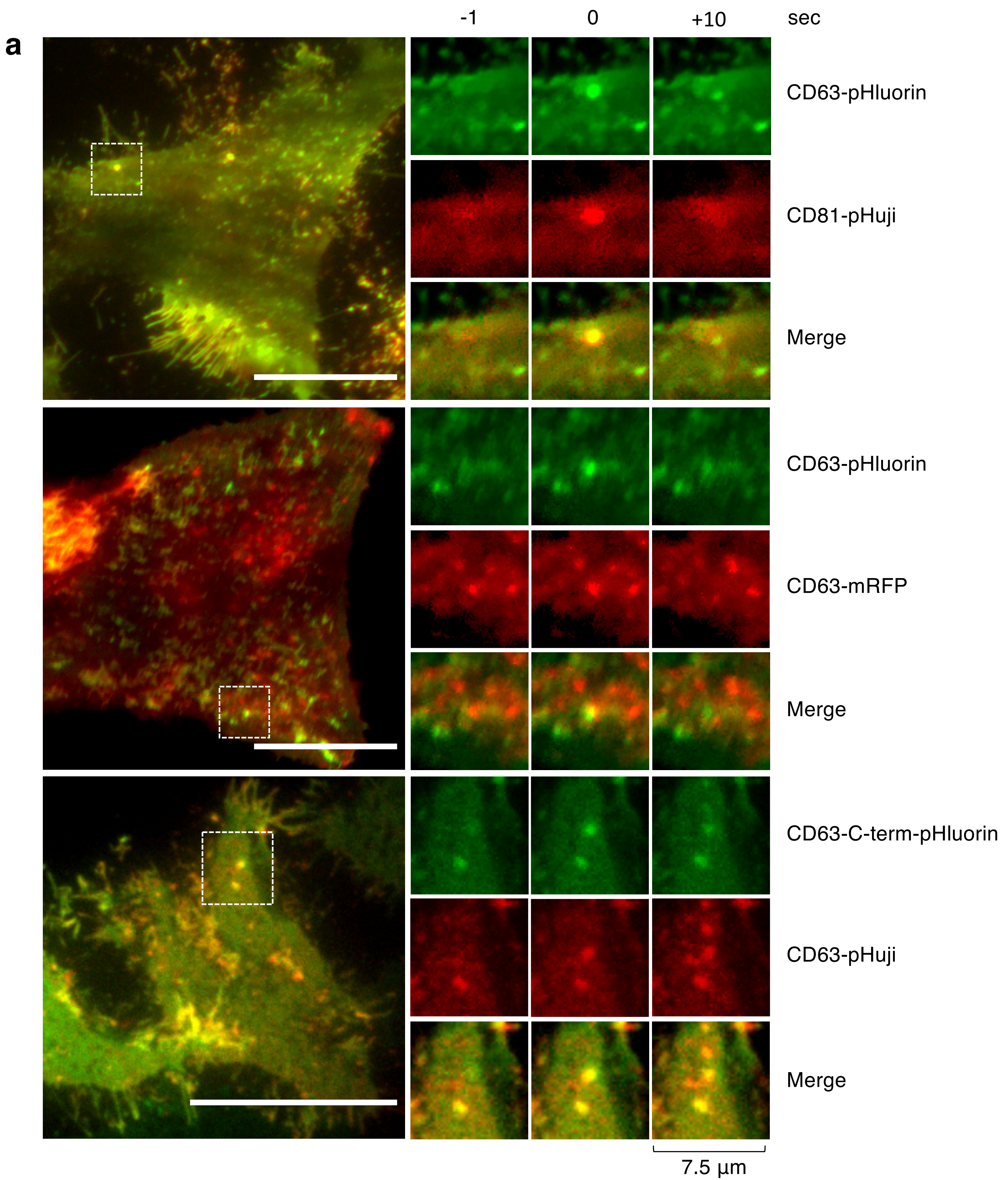

b

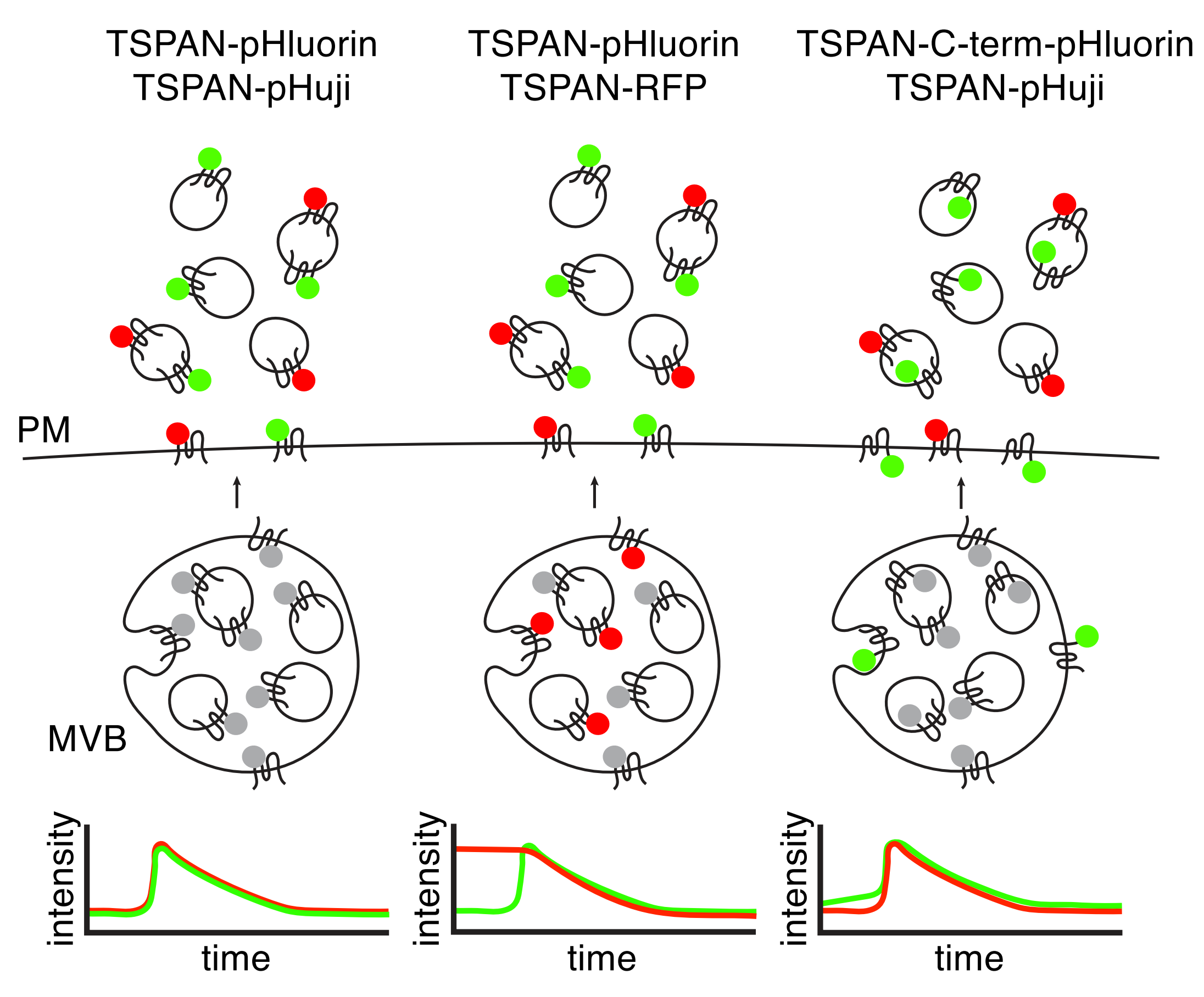

C

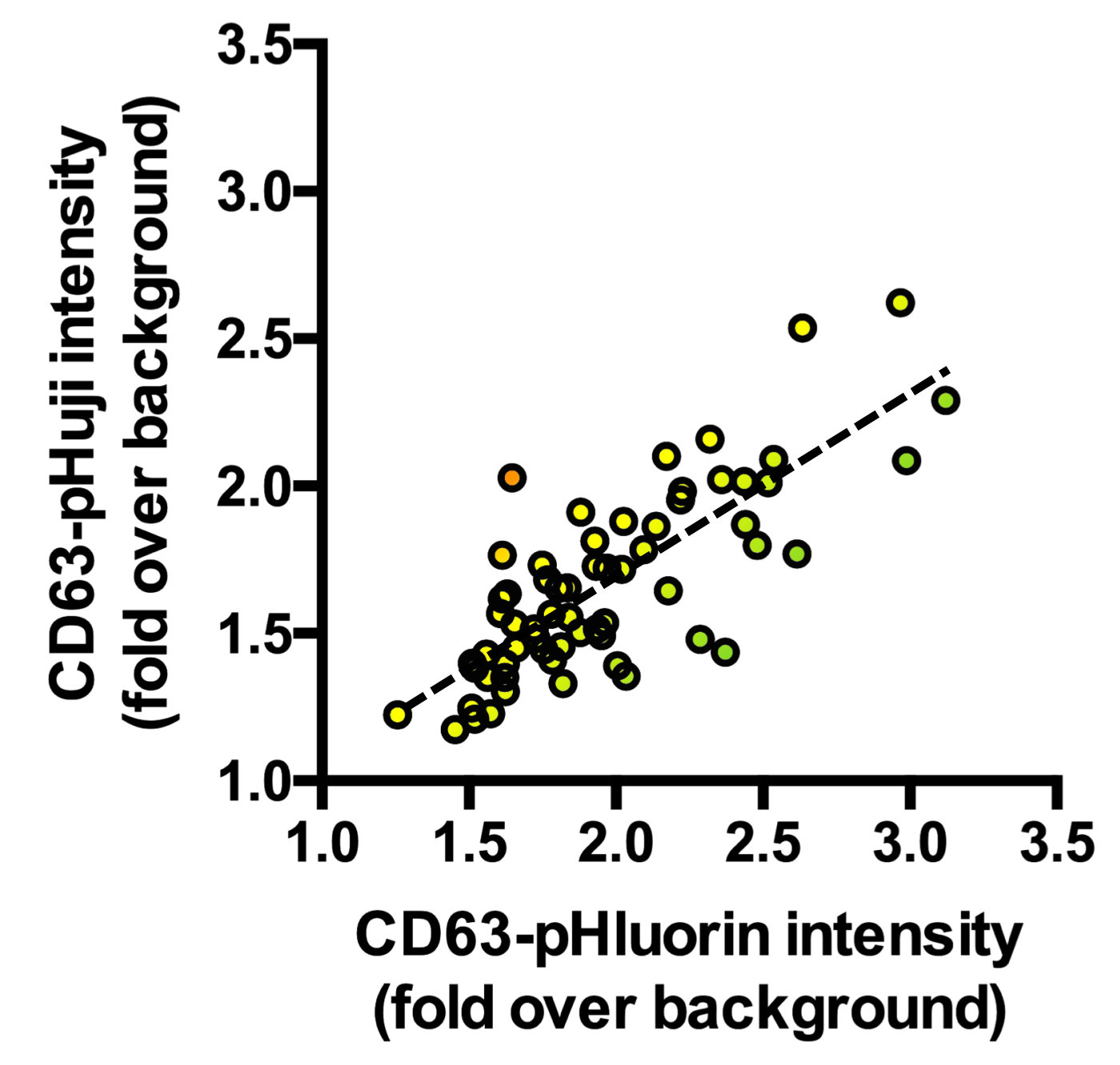



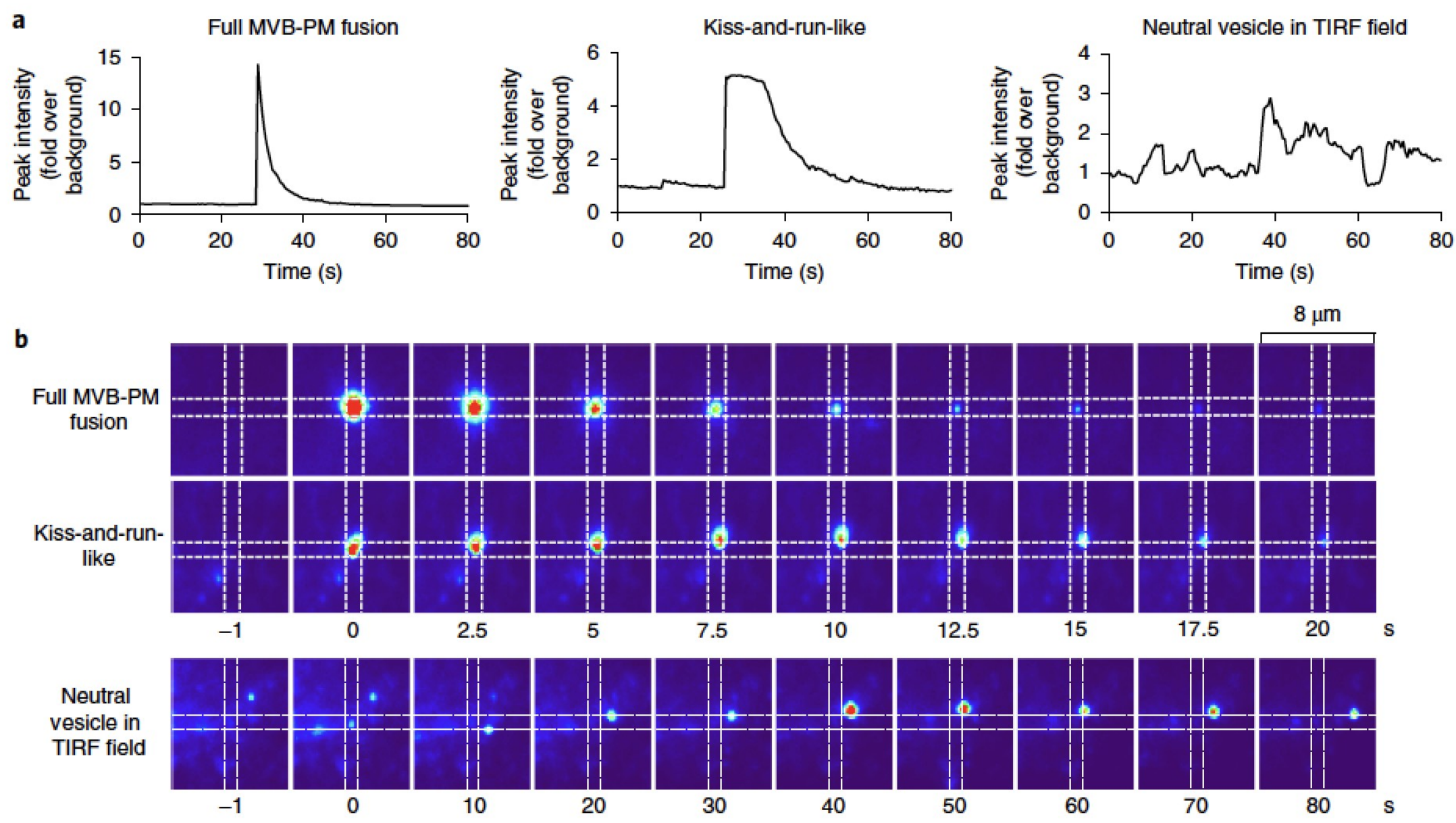

c
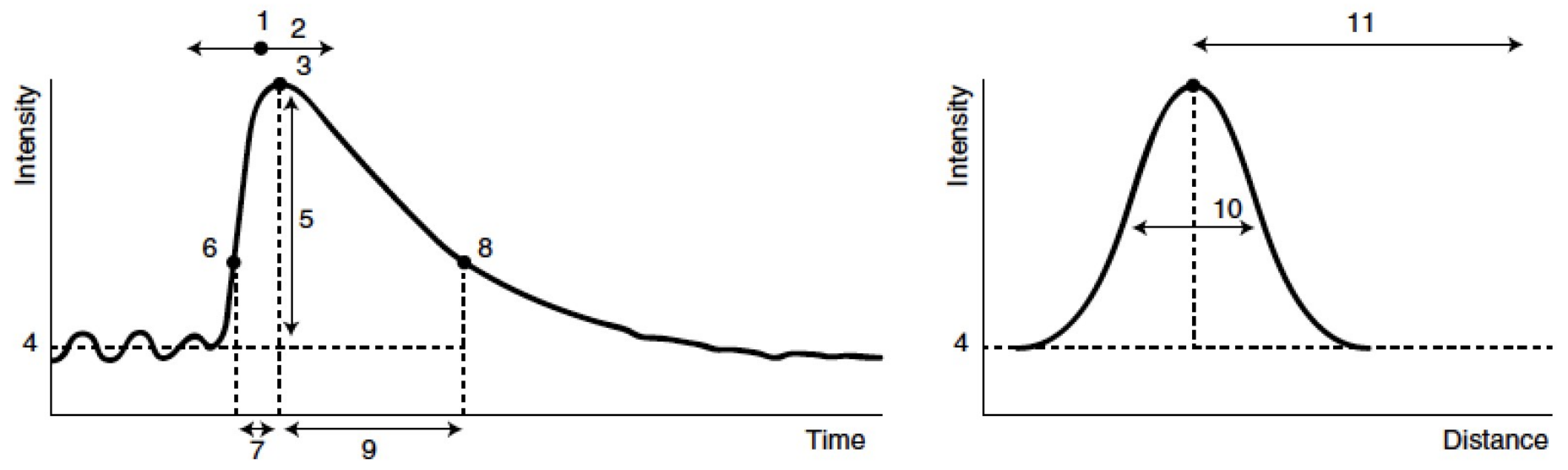


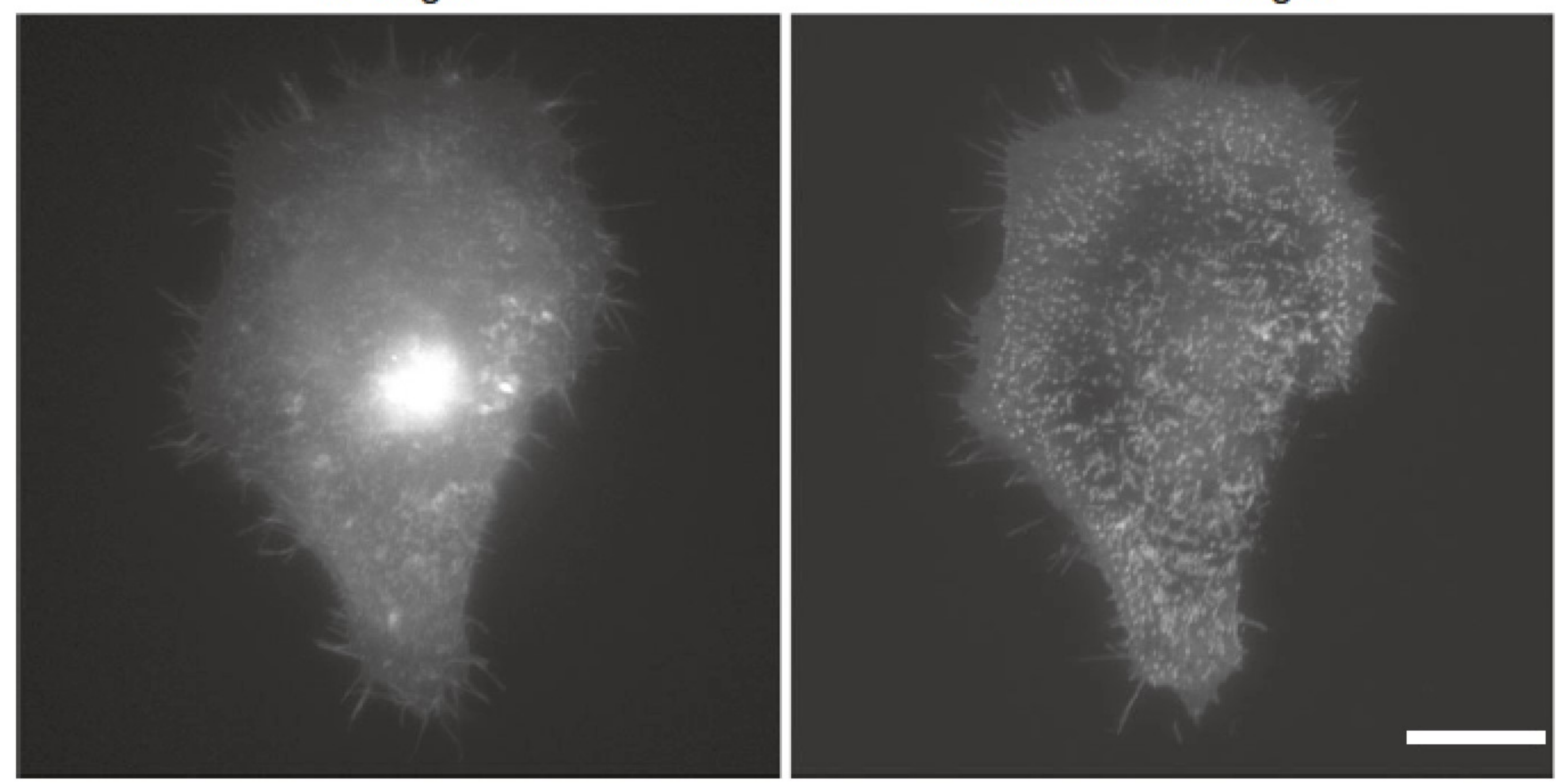


Fig 5

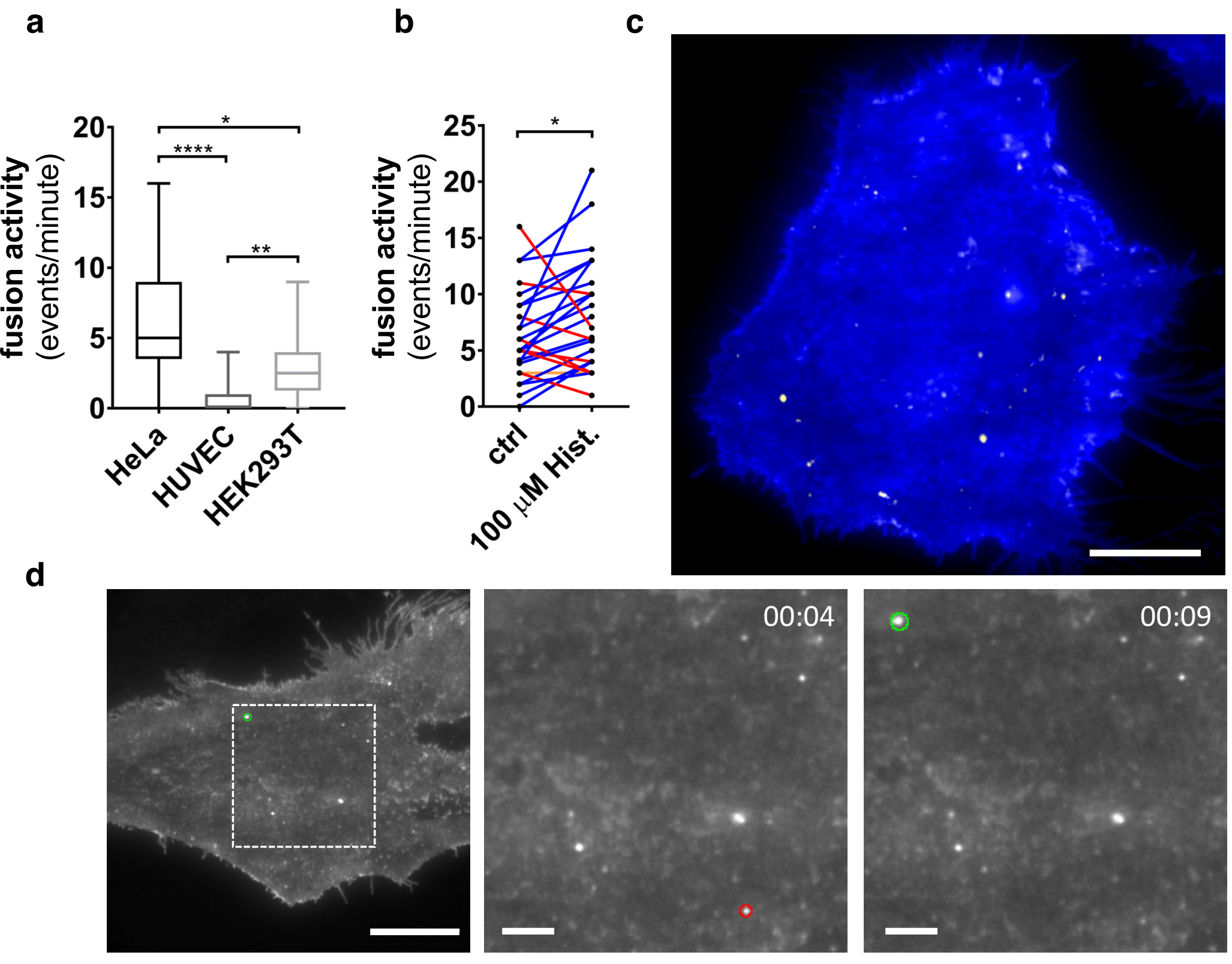

